\title{
Antioxidants as Adjuvants in Periodontitis Treatment: A Systematic Review and Meta-Analysis
}

\author{
Micaele Maria Lopes Castro, ${ }^{1}$ Nathallia Neves Duarte, ${ }^{1}$ Priscila Cunha Nascimento, ${ }^{1}$ \\ Marcela Barauna Magno, ${ }^{2}$ Nathalia Carolina Fernandes Fagundes, ${ }^{1,3}$ Carlos Flores-Mir, ${ }^{3}$ \\ Marta Chagas Monteiro $\mathbb{D}^{4}$, Cassiano Kuchenbecker Rösing, ${ }^{5}$ Lucianne Cople Maia $\mathbb{D}^{2}{ }^{2}$ \\ and Rafael Rodrigues Lima $\mathbb{B D}^{1}$ \\ ${ }^{1}$ Laboratory of Functional and Structural Biology, Institute of Biological Sciences, Federal University of Pará, Belém, Brazil \\ ${ }^{2}$ Department of Pediatric Dentistry and Orthodontics, School of Dentistry, Federal University of Rio de Janeiro, Rio de Janeiro, Brazil \\ ${ }^{3}$ School of Dentistry, Faculty of Medicine and Dentistry, University of Alberta, Edmonton, Canada \\ ${ }^{4}$ School of Pharmacy, Federal University of Pará, Belém, Pará, Brazil \\ ${ }^{5}$ Department of Periodontology, Faculty of Dentistry, Federal University of Rio Grande do Sul, Porto Alegre, Brazil
}

Correspondence should be addressed to Rafael Rodrigues Lima; rafalima@ufpa.br

Received 10 March 2019; Revised 18 May 2019; Accepted 3 June 2019; Published 22 July 2019

Guest Editor: MARILIA Goulart

Copyright (c) 2019 Micaele Maria Lopes Castro et al. This is an open access article distributed under the Creative Commons Attribution License, which permits unrestricted use, distribution, and reproduction in any medium, provided the original work is properly cited.

\begin{abstract}
This systematic review with meta-analysis aimed to evaluate the effect of antioxidants as an adjuvant in periodontitis treatment. The following databases were consulted: PubMed, Scopus, Web of Science, Cochrane, Lilacs, OpenGrey, and Google Scholar. Based on the PICO strategy, the inclusion criteria comprised interventional studies including periodontitis patients (participants) treated with conventional therapy and antioxidants (intervention) compared to patients treated only with conventional therapy (control) where the periodontal response (outcome) was evaluated. The risk of bias was evaluated using the Cochrane RoB tool (for randomized studies) and ROBINS-I tool (for nonrandomized studies). Quantitative data were analyzed in five random effects meta-analyses considering the following periodontal parameters: clinical attachment loss (CAL), plaque index (PI), gingival index (GI), bleeding on probing (BOP), and probing depth (PD). After all, the level of certainty was measured with the Grading of Recommendation, Assessment, Development, and Evaluation (GRADE) tool. Among the 1884 studies identified, only 15 interventional studies were according to the eligibility criteria and they were included in our review. From them, 4 articles presented a high risk of bias. The meta-analysis showed a statistically significant difference for CAL $\left(\operatorname{SMD} 0.29(0.04,0.55), p=0.03, I^{2}=13 \%\right)$, PI (SMD $\left.0.41(0.18,0.64), p=0.0005, I^{2}=47 \%\right)$, and BOP (SMD 0.55 (0.27, 0.83$)$, $\left.p=0.0001, I^{2}=0 \%\right)$. The GRADE tool showed a moderate to high certainty in the quality of evidence depending on the clinical parameter and antioxidants used. These results suggest that the use of antioxidants is an adjunct approach to nonsurgical periodontal therapy which may be helpful in controlling the periodontal status.
\end{abstract}

\section{Introduction}

Periodontal disease is a chronic inflammatory manifestation in the tissues surrounding the teeth caused by an imbalance between oral biofilms and the host's response, in which there is a possibility of loss of tooth support tissues $[1,2]$. The state of being restricted to protective periodontium is characterized as gingivitis, and that when affecting the periodontal supporting tissues is called periodontitis [3]. Periodontitis is one of the most prevalent diseases of the oral cavity, caused by bacterial plaque microorganisms and influenced by factors such as systemic condition, oral hygiene, age, sex, and smoking [4].

The excessive presence of free radicals caused by oxidative stress or antioxidant deficiency has been linked to periodontal disease [5]. Early in the progression of periodontal 
disease, especially in periodontitis, there is a marked oxidative process with increased levels of reactive oxygen and nitrogen species (ROS and RNS). This process can lead to an imbalance in the body response, with concomitant changes in biomolecules, especially lipids, proteins, and nucleic acids, resulting in periodontal tissue damage [6].

The antioxidant defense system can inhibit and/or reduce the damage caused by deleterious action of free radicals or nonradical reactive species [7]. Some antioxidant sources found in nature (foods, teas, vitamins, minerals, among others) are currently used in various auxiliary treatments for cardiovascular diseases, pulmonary diseases, ageing, and atherosclerosis [8-11]. These conditions have physiologic links with periodontal diseases which would lead to the assumptions that a potential benefit could also be observed under these therapies.

In the search for adjuvants to conventional periodontal treatments which could present worse than expected results, some literature suggests that supplementation with antioxidant components may help to reduce periodontal damage and its systemic effects when compared to treatment with antibiotics that can cause resistance or onset of secondary infections [12]. There is not necessarily a consensus in this regard, as the evidence regarding the supporting role of antioxidant agents as adjuvants to periodontal treatment is limited which makes clinical decision-making difficult. Therefore, this systematic review is aimed at assessing whether antioxidants have some beneficial effect on the treatment of periodontitis.

\section{Material and Methods}

2.1. Focus Question and Registration. In order to perform this systematic review, the following question was elaborated: "In patients with periodontitis, do antioxidants have an additional clinically meaningful effect when used as adjuvants to conventional therapy?"

This systematic review was registered under the number CRD42017079869 in the PROSPERO database, created by the University of York, responsible for the registration and dissemination of systematic reviews and carried out according to the PRISMA (Preferred Reporting Items for Systematic Reviews and Meta-Analyses) statement [13].

\subsection{Search Strategy and Eligibility Criteria. The PICO strat-} egy was applied in this systematic review. This acronym stands for an abbreviation of the following components: patient, intervention, comparison, and outcome, which are essential for designing all stages of an interventional systematic review. We included intervention studies in adult humans with periodontitis $(\mathrm{P})$ that compared conventional periodontal treatment with the addition of antioxidants (I) compared to patients who have undergone only conventional periodontal treatment $(\mathrm{C})$ in order to observe periodontal treatment effects (O). Additionally, case reports, descriptive studies, review articles, opinion articles, technical articles, guidelines, animal studies, and in vitro studies were not considered.
The electronic searches were carried out in the following databases: PubMed, Scopus, Web of Science, Lilacs, Cochrane, Google Scholar, and OpenGrey. There were no restrictions on the date of publication or in the language used in the primary studies. The terms $\mathrm{MeSH}$, keywords, and search strategies were adapted according to each database (Table 1). The searches were carried out up to February 2019.

A search alert was created in each database to notify new studies according to the outlined search strategy. All relevant citations were imported into a bibliographic reference manager (EndNote ${ }^{\circledR}$, version X7, Thomson Reuters, Philadelphia, USA).

Through the EndNote ${ }^{\circledR}$ manager, the removal of duplicate articles was performed using automatic exclusion added to the manual deletion. After the exclusions, the texts were analyzed for the titles and abstracts and, afterwards, from the reading of the full text when indicated, according to the established eligibility criteria.

2.3. Data Extraction and Quality Assessment. The eligible articles resulting from the previous selection were extracted and tabulated including information of the country, year, characteristics of the participants (sample size and age), periodontal parameters measured, the antioxidant used, conventional treatment performed, results, and statistical analysis.

The risk of bias assessment was based on two tools. "The Cochrane Collaboration's tool for assessing risk of bias" tool [14] through the Review Manager software (version 5.3, Review Manager (RevMan) (computer program) version 5.3. Copenhagen: The Nordic Cochrane Centre, The Cochrane Collaboration, 2014) was used for randomized intervention studies and the ROBINS-I (Risk of Bias in Non-randomized Studies of Interventions) tool [15] for nonrandomized studies.

“The Cochrane Collaboration's tool for assessing risk of bias" tool [14] consists of a checklist of seven key areas: sequence generation randomized, concealment selection, blinding of participants, evaluation of blinding result, incomplete result data, selective reporting bias, and other risks (Table 2). For each study, the risk of bias was judged for each domain and the overall assessment as low risk, high risk, or uncertain risk for all included studies.

The ROBINS-I (Risk of Bias in Non-randomized Studies of Interventions) tool [15] is composed of three main domains for bias evaluation: preintervention, during intervention, and postintervention (Table 3 ). The risk of bias was judged for each domain and classified in its general assessment as low, moderate, serious, critical, or no information for all included studies.

All evaluations, including searches, study selection, and data extraction, were performed independently by two reviewers (NND and MMLC) and checked by a third-party evaluator (RRL) in case of disagreement.

2.4. Meta-Analysis. Data from the included studies were analyzed using Comprehensive Meta-Analysis software (version 3.2; Biostat) to evaluate the efficacy of periodontal therapy (PT) associated with antioxidants in periodontal parameters. This treatment protocol (PT plus antioxidants) was compared 
TABLE 1: Search strategy on each electronic database.

\begin{tabular}{|c|c|}
\hline Database & Search format \\
\hline PubMed & 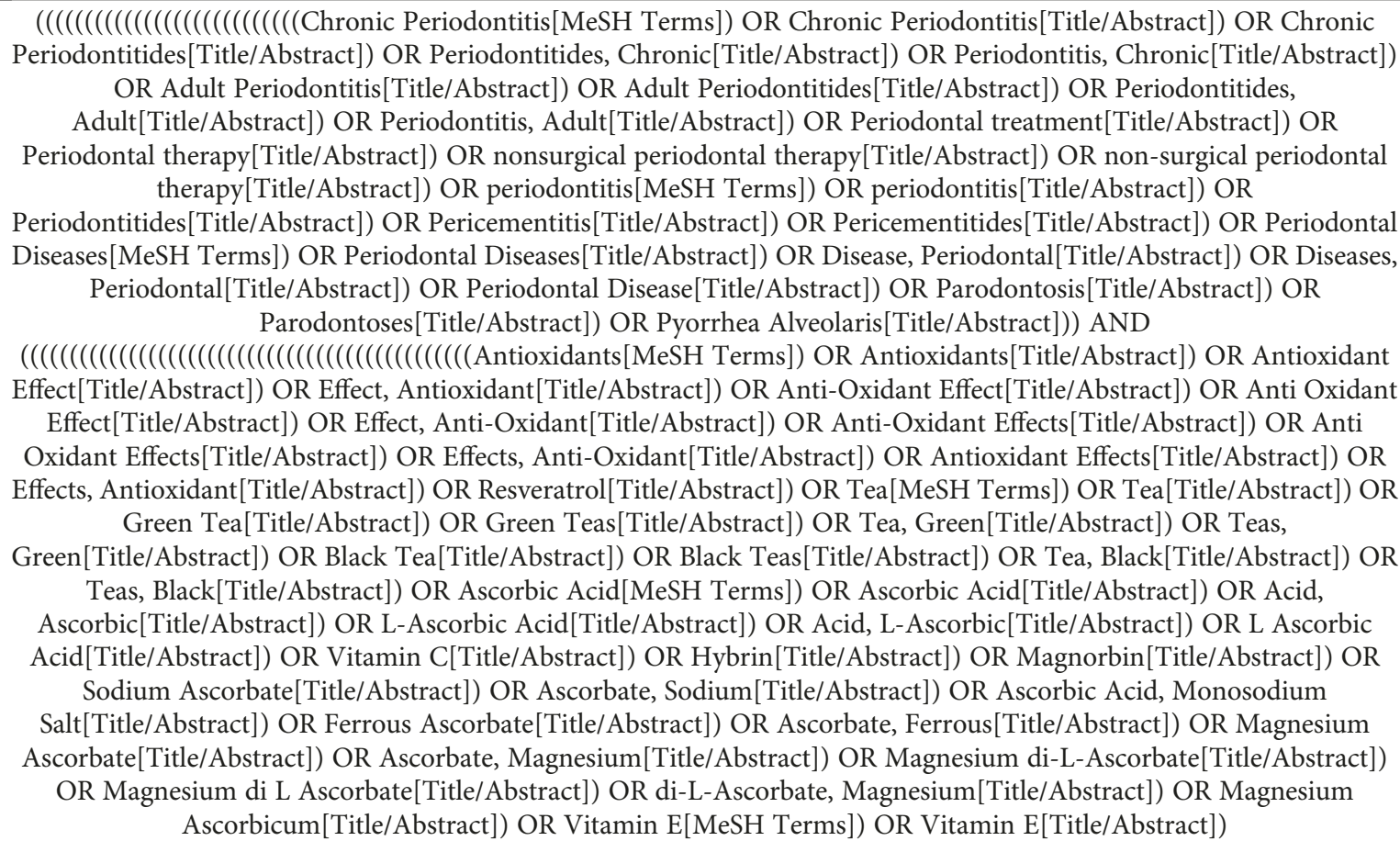 \\
\hline
\end{tabular}

(TITLE-ABS-KEY (“Chronic Periodonti*”) OR TITLE-ABS-KEY (“Periodontitides, Chronic”) OR TITLE-ABS-KEY

(“Periodontitis, Chronic”) OR TITLE-ABS-KEY (“Adult Periodontiti*”) OR TITLE-ABS-KEY ("Periodontitides, Adult”)

OR TITLE-ABS-KEY (“Periodontitis, Adult”) OR TITLE-ABS-KEY (“Periodontal treatment”) OR TITLE-ABS-KEY

("Periodontal therapy") OR TITLE-ABS-KEY ("nonsurgical periodontal therapy") OR TITLE-ABS-KEY ("non-surgical periodontal therapy”) OR TITLE-ABS-KEY (Periodontiti*) OR TITLE-ABS-KEY (Pericementiti*) OR TITLE-ABS-KEY

(“Periodontal Disease*”) OR TITLE-ABS-KEY (“Disease, Periodontal”) OR TITLE-ABS-KEY (“Diseases, Periodontal”)

OR TITLE-ABS-KEY ("Parodontos*”) OR TITLE-ABS-KEY ("Pyorrhea Alveolaris")) AND (TITLE-ABS-KEY

(Antioxidants) OR TITLE-ABS-KEY (“Antioxidant Effect*”) OR TITLE-ABS-KEY (“Effect, Antioxidant”) OR

TITLE-ABS-KEY (“Anti-Oxidant Effect*”) OR TITLE-ABS-KEY (“Anti Oxidant Effect*”) OR TITLE-ABS-KEY (“Effect,

Anti-Oxidant”) OR TITLE-ABS-KEY ("Effects, Anti-Oxidant”) OR TITLE-ABS-KEY ("Effects, Antioxidant”) OR
Scopus
TITLE-ABS-KEY (Resveratrol) OR TITLE-ABS-KEY (Tea) OR TITLE-ABS-KEY ("Green Tea*") OR TITLE-ABS-KEY

(“Tea, Green”) OR TITLE-ABS-KEY (“Teas, Green”) OR TITLE-ABS-KEY (“Black Tea*”) OR TITLE-ABS-KEY

(“Tea, Black”) OR TITLE-ABS-KEY (“Teas, Black”) OR TITLE-ABS-KEY (“Ascorbic Acid”) OR TITLE-ABS-KEY

(“Acid, Ascorbic”) OR TITLE-ABS-KEY (“L-Ascorbic Acid”) OR TITLE-ABS-KEY (“Acid, L-Ascorbic”) OR TITLE-ABSKEY ("L Ascorbic Acid”) OR TITLE-ABS-KEY ("Vitamin C”) OR TITLE-ABS-KEY (Hybrin) OR TITLE-ABS-KEY (Magnorbin) OR TITLE-ABS-KEY (“Sodium Ascorbate”) OR TITLE-ABS-KEY (“Ascorbate, Sodium”) OR TITLE-ABSKEY ("Ascorbic Acid, Monosodium Salt ") OR TITLE-ABS-KEY ("Ferrous Ascorbate”) OR TITLE-ABS-KEY ("Ascorbate, Ferrous”) OR TITLE-ABS-KEY (“Magnesium Ascorbate”) OR TITLE-ABS-KEY (“Ascorbate, Magnesium”) OR TITLEABS-KEY ("Magnesium di-L-Ascorbate") OR TITLE-ABS-KEY ("Magnesium di L Ascorbate") OR TITLE-ABS-KEY (“di-L-Ascorbate, Magnesium”) OR TITLE-ABS-KEY (“Magnesium Ascorbicum”) OR TITLE-ABS-KEY (“Vitamin E”))

TS=(“Chronic Periodonti*”) OR TS=(“Periodontitides, Chronic”) OR TS=(“Periodontitis, Chronic") OR TS=(“Adult Periodontiti*”) OR TS=("Periodontitides, Adult") OR TS=("Periodontitis, Adult") OR TS=("Periodontal treatment") OR TS=("Periodontal therapy") OR TS=("nonsurgical periodontal therapy") OR TS=("non-surgical periodontal therapy") OR TS=(Periodontiti $*)$ OR TS=(Pericementiti $*)$ OR TS=(“Periodontal Disease $*$ ”) OR TS=(“Disease, Periodontal”) OR TS=("Diseases, Periodontal") OR TS=("Parodontos*”) OR TS=("Pyorrhea Alveolaris") AND TS=(Antioxidants) OR TS=(“Antioxidant Effect*”) OR TS=(“Effect, Antioxidant”) OR TS=(“Anti-Oxidant Effect*”) OR TS=(“Anti Oxidant

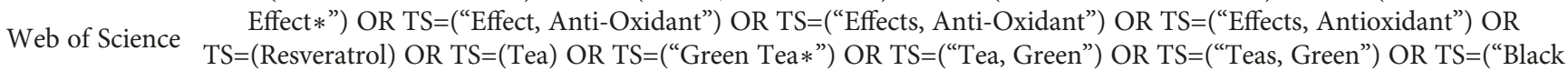
Tea*”) OR TS=(“Tea, Black”) OR TS=(“Teas, Black”) OR TS=(“Ascorbic Acid”) OR TS=(“Acid, Ascorbic”) OR TS=("L-Ascorbic Acid") OR TS=(“Acid, L-Ascorbic") OR TS=("L Ascorbic Acid”) OR TS=("Vitamin C") OR TS=(Hybrin) OR TS=(Magnorbin) OR TS=("Sodium Ascorbate”) OR TS=("Ascorbate, Sodium”) OR TS=("Ascorbic Acid, Monosodium Salt") OR TS=("Ferrous Ascorbate") OR TS=("Ascorbate, Ferrous") OR TS=("Magnesium Ascorbate") OR TS=(“Ascorbate, Magnesium”) OR TS=("Magnesium di-L-Ascorbate") OR TS=("Magnesium di L Ascorbate") OR TS=(“di-L-Ascorbate, Magnesium") OR TS=("Magnesium Ascorbicum") OR TS=("Vitamin E”) 
TABle 1: Continued.

\begin{tabular}{|c|c|}
\hline Database & Search format \\
\hline Cochrane & 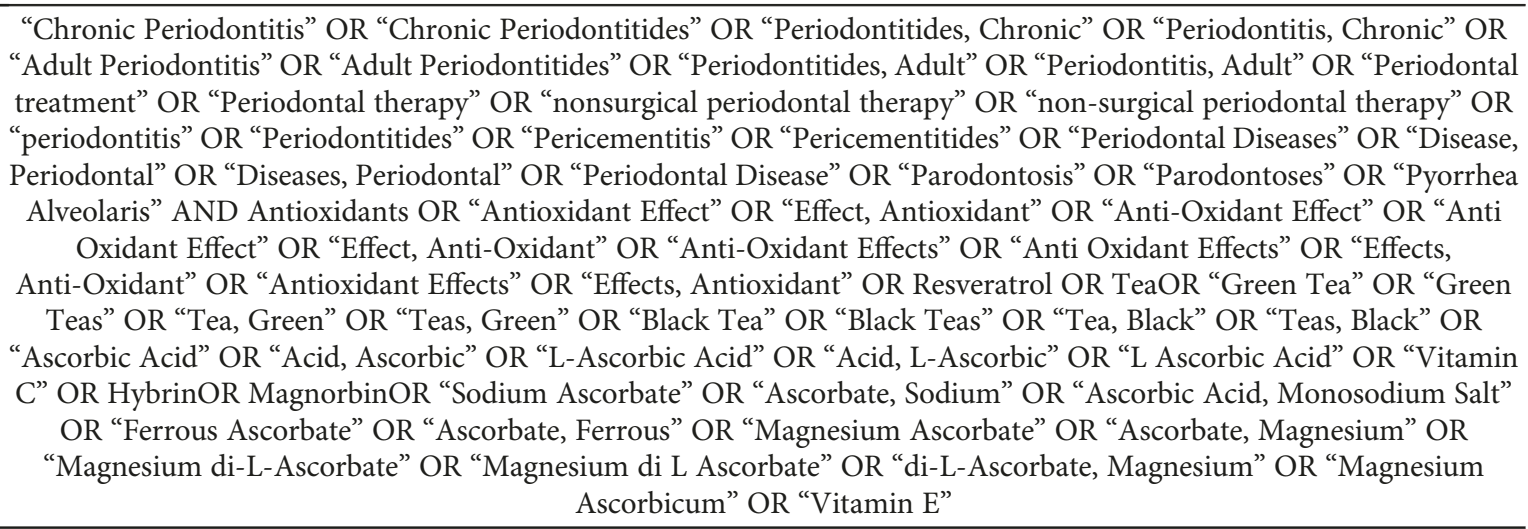 \\
\hline Lilacs & $\begin{array}{l}\text { (tw:((Chronic Periodontitis) OR (Chronic Periodontitides) OR (Periodontitides, Chronic) OR (Periodontitis, Chronic) OR } \\
\text { (Adult Periodontitis) OR (Adult Periodontitides) OR (Periodontitides, Adult) OR (Periodontitis, Adult) OR (Periodontal } \\
\text { treatment) OR (Periodontal therapy) OR (nonsurgical periodontal therapy) OR (non-surgical periodontal therapy) OR } \\
\text { (periodontitis) OR (Periodontitides) OR (Pericementitis) OR (Pericementitides) OR (Periodontal Disease\$) OR (Disease, } \\
\text { Periodontal) OR (Diseases, Periodontal) OR (Parodontosis) OR (Parodontoses) OR (Pyorrhea Alveolaris))) AND } \\
\text { (tw:((Antioxidant\$) OR (Antioxidant Effect) OR (Effect, Antioxidant) OR (Anti-Oxidant Effect) OR (Anti Oxidant Effect) } \\
\text { OR (Effect, Anti-Oxidant) OR (Effects, Anti-Oxidant) OR (Effects, Antioxidant) OR (Resveratrol) OR (Tea\$) OR (Green } \\
\text { Tea\$) OR (Tea, Green) OR (Teas, Green) OR (Black Tea\$) OR (Tea, Black) OR (Teas, Black) OR (Ascorbic Acid) OR } \\
\text { (Acid, Ascorbic) OR (L-Ascorbic Acid) OR (Acid, L-Ascorbic) OR (L Ascorbic Acid) OR (Vitamin C) OR (Hybrin) OR } \\
\text { (Magnorbin) OR (Sodium Ascorbate) OR (Ascorbate, Sodium) OR (Ascorbic Acid, Monosodium Salt) OR (Ferrous } \\
\text { Ascorbate) OR (Ascorbate, Ferrous) OR (Magnesium Ascorbate) OR (Ascorbate, Magnesium) OR (Magnesium } \\
\text { di-L-Ascorbate) OR (Magnesium di L Ascorbate) OR (di-L-Ascorbate, Magnesium) OR (Magnesium Ascorbicum) } \\
\text { OR (Vitamin E))) }\end{array}$ \\
\hline Google Scholar & Periodontitis+ Antioxidants -review \\
\hline OpenGrey & Periodontitis AND Antioxidants \\
\hline
\end{tabular}

to conventional PT only. The main periodontal parameters analyzed by the studies were evaluated in five different random effects meta-analyses: $\left(1^{\text {st }}\right)$ clinical attachment loss $(\mathrm{CAL}),\left(2^{\text {nd }}\right)$ plaque index $(\mathrm{PI}),\left(3^{\mathrm{rd}}\right)$ gingival index $(\mathrm{GI})$, $\left(4^{\text {th }}\right)$ bleeding on probing (BOP), and $\left(5^{\text {th }}\right)$ probing depth (PD). The average and standard deviation of each parameter and the total number of individuals of each group (PT only and PT plus antioxidants) were used. A subgroup analysis was conducted considering the follow-up periods evaluated in studies.

As the studies reported the outcome using similar methods for all parameters, the standard mean difference (SMD) was applied [16], with 95\% confidence interval (CI). Only studies considered as having "low," "unclear," and "moderate" risks of bias were included in the meta-analysis. If some of the information needed for the meta-analysis was absent from any of the selected studies, the authors were contacted to provide the missing data. Studies considered as having a high risk of bias and/or remaining without sufficient data for the quantitative analysis, after contact with the authors, were excluded from the meta-analysis.

Heterogeneity was tested using the $I^{2}$ index, and if possible, sensitivity analyses were conducted to estimate and verify the influence of studies, one by one, on the pooled results, when the heterogeneity was substantial or considerable (50 to 100\%) [16]. Random effects models were employed taking into consideration that the studies were not functionally equivalent in which the objective was to generalize the results from the meta-analysis [17].

2.5. Level of Evidence. A summary of the overall strength of evidence was presented using the "Grading of Recommendation, Assessment, Development, and Evaluation" (GRADE) tool [18]. Evidence from randomized clinical trials is initially classified as high quality, but the assurance on this evidence may be reduced for a number of reasons including the following: the methodological design, study quality, consistency, and directness. Three subgroups were created dividing according to the antioxidants used: (1) several antioxidants; (2) lycopene; (3) green tea.

\section{Results}

3.1. Characteristics of the Included Studies. The searches in databases and gray literature articles amounted to 3213, resulting in 1884 after exclusion of duplicates. All 1884 remaining articles were analyzed by titles and abstracts, based on the eligibility criteria, and then 1831 were excluded. Thirty-six studies were analyzed in full text, and 21 of them were excluded, having 15 articles remaining that were included in this review to qualitative synthesis [19-33], 
TABLE 2: Criteria for risk assessment of bias according to "the Cochrane Collaboration's tool for assessing risk of bias (Higgins et al., 2011).

\begin{tabular}{l}
$\begin{array}{l}\text { Random sequence generation } \\
\text { Criteria for judgment of "low risk" of bias }\end{array}$ \\
$\begin{array}{l}\text { The articles that appropriately described the method of randomization } \\
\text { Criteria for judgment of "high risk" of bias }\end{array}$ \\
$\begin{array}{c}\text { Criteria for judgment of "unclear risk" of bias } \\
\text { or the difficult reproducibility method }\end{array}$ \\
\hline
\end{tabular}

Allocation concealment

Criteria for judgment of "low risk" of bias

When the allocation sequences of samples were concealed in the randomization

Criteria for judgment of "high risk" of bias

Criteria for judgment of "unclear risk" of bias

When the sequences of allocation of samples were not concealed at randomization When the allocation sequences were unreported

Blinding of participants and researchers

Criteria for judgment of "low risk" of bias

When the sample was blind

Criteria for judgment of "high risk" of bias

Criteria for judgment of "unclear risk" of bias

Blinding of outcome assessment

Criteria for judgment of "low risk" of bias

Criteria for judgment of "high risk" of bias

Criteria for judgment of "unclear risk" of bias

Incomplete outcome data

Criteria for judgment of "low risk" of bias

When there was an exhaustive description of the main data

Criteria for judgment of "high risk" of bias

Criteria for judgment of "unclear risk" of bias

Selective reporting

Criteria for judgment of "low risk" of bias

Criteria for judgment of "high risk" of bias

Criteria for judgment of "unclear risk" of bias

When the evaluators reported that the blinding in the evaluation was effective If the study informed the evaluators how the blinding was done When the blinding was not reported

If the methodology could not be blinded for whatever reason (sample/appraiser)

When the sample was not reported either way

and 7 of them were designated to quantitative synthesis $[19,22,23,25,28,30,32]$ (Figure 1).

All studies evaluated periodontitis, and in those where there was a group for gingivitis and group for periodontitis presented in the same study $[22,29]$, only data referring to periodontitis were considered. Authors, study design, sample description, periodontitis diagnostic method, type of periodontal and antioxidant treatment, statistical analysis, and the outcome of each included article were described in Table 4.

The PD was the most used evaluation method, present in all studies, followed by gingival and plaque indexes (GI and $\mathrm{PI}$, respectively), CAL, and BOP. One study reports the additional use of the community periodontal index (CPI) as an evaluation method [28]. Regarding periodontitis treatment, all studies carried out scaling and root planning; however, only one study added a surgical treatment, the modified Widman flap procedure, specifically at baseline visit.

Some studies have evaluated laboratory aspects besides clinical aspects such as markers of bone resorption (RANKL) [19], markers of inflammatory response (interleukins, tumor necrosis factor alpha, and Pentraxin-3) [20, 24, 26], nitrite/nitrate ratio, and antioxidant activity markers (total antioxidant capacity, glutathione-S-transferase, uric acid, and superoxide dismutase) $[21,23,25,29,31,32]$, present in saliva, crevicular fluid, or plasma. Only five studies did not perform laboratory tests [22, 27, 28, 30, 33].

3.2. Risk of Bias. A total of 14 randomized clinical trials were assessed for bias risk using the Cochrane tool [14]. The key domains chosen for the high-risk bias trial were allocation concealment, blinding of participants and personnel, and blinding of outcome assessment. From them, 11 studies showed a low risk of bias while 4 studies demonstrated a high risk of bias from the evaluation proposed by the key domains chosen [24, 26, 27, 33] (Figure 2).

In the risk of bias analysis in the ROBINS-I model, the study by Mathur et al. [29] presented a low risk of bias in almost all domains, except in the bias of missing data, because it did not present enough data where some judgment could be attributed (Table 5).

3.3. Meta-Analysis. Four studies were excluded from the meta-analyses because they were classified as having "high" risk of bias [24, 26, 27, 33]. Data extracted by the Singh et al. study were imprecise, and for this reason, this study was excluded from the quantitative synthesis. The metaanalysis results were presented separately for each parameter.

3.3.1. Meta-Analysis for Clinical Attachment Loss (CAL). Seven clinical trials evaluating the influence of PT 
TABLE 3: Risk of bias evaluation of nonrandomized clinical trials according to the ROBINS-I tool [15].

Domain of bias
$\begin{aligned} & \text { Preintervention } \\ & \text { Bias due to confounding }\end{aligned} \begin{array}{r}\text { Description } \\ \text { Baseline confounding. When one or more preintervention prognostic factors predict th } \\ \text { intervention received at baseline (start of follow-up) } \\ \text { Time-varying confounding. When the intervention received can change over time and } \\ \text { when postintervention prognostic factors affect the intervention received after baseline } \\ \text { When selection of participants is related to both intervention and outcome } \\ \text { Lead time bias. When some follow-up time is excluded from the analysis } \\ \text { Bias in selecting participants for study }\end{array}$
$\begin{array}{r}\text { Immortal time bias. When the interventions are defined in such a way that there is a } \\ \text { period of follow-up during which the outcome cannot occur }\end{array}$

At intervention

Bias in classifying interventions

When intervention status is misclassified

Nondifferential misclassification. Is unrelated to the outcome

Differential misclassification. Is related to the outcome or to the risk of the outcome

Postintervention

Bias due to deviating from intended intervention

Bias due to missing data

Bias in measuring outcomes

Bias in selecting reported result
When there are systematic differences between intervention and comparator groups in the care provided

When attrition (loss to follow-up), missed appointments, incomplete data collection, and exclusion of participants from analysis by primary investigators occur

When outcomes are misclassified or measured with error

Nondifferential measurement error. Is unrelated to the intervention received; it can be systematic or random

Selective reporting of results that should be sufficiently reported to allow the estimate to be included in a meta-analysis (or other synthesis) is considered. When selective reporting is based on the direction, magnitude, or statistical significance of intervention effect estimates. Selective outcome reporting. When the effect estimate for an outcome measurement was selected from among analyses of multiple outcome measurements for

the outcome domain. Selective analysis reporting. When results are selected from intervention effects estimated in multiple ways

Judgment for each domain

Low RoB

Study is comparable to a well-performed, randomized trial with regard to this domain

Moderate RoB

Study is sound for a nonrandomized study with regard to this domain but cannot be considered comparable to a well-performed, randomized trial

Study has some important problems in this domain

Serious RoB

Critical RoB

Study is too problematic in this domain to provide any useful evidence on the effects of intervention

No information

No information on which to base a judgment about risk of bias for this domain

Overall judgment

Low RoB

Moderate RoB

Serious RoB

Critical RoB

No information
Study is judged to be at low risk of bias for all domains

Study is judged to be at low or moderate risk of bias for all domains

Study is judged to be at serious risk of bias in at least one domain, but not at critical risk of bias in any domain

Study is judged to be at critical risk of bias in at least one domain

No clear indication that the study is at serious or critical risk of bias, and there is a lack of information in one or more key domains of bias (a judgment is required for this) additionally to antioxidant therapy in the mean of CAL were included in this analysis. Including all studies, the heterogeneity was $I^{2}=61 \%$. During sensitivity analysis, the heterogeneity ranges from $20 \%$ to $66 \%$, and in an attempt to reduce the overall and subgrouped heterogeneity, the study of Chopra et al. [23] was excluded from the final analysis. In subgroup analysis, independent of follow-up point evaluated, individuals treated with PT plus antioxidants presented a mean CAL, in millimeters, similar to individuals treated with PT only (up to one month (SMD $0.38(-0.02,0.78), p=0.06$, $\left.I^{2}=21 \%, I^{2} p=0.28\right)$, three months (SMD $0.28(-0.18,0.73)$, $\left.p=0.23, I^{2}=15 \%, I^{2} p=0.31\right)$, or six months or more (SMD $\left.0.22(-0.62,1.05), p=0.61, I^{2}=58 \%, I^{2} p=0.12\right)$ ). However, in pooled results, individuals treated with PT 


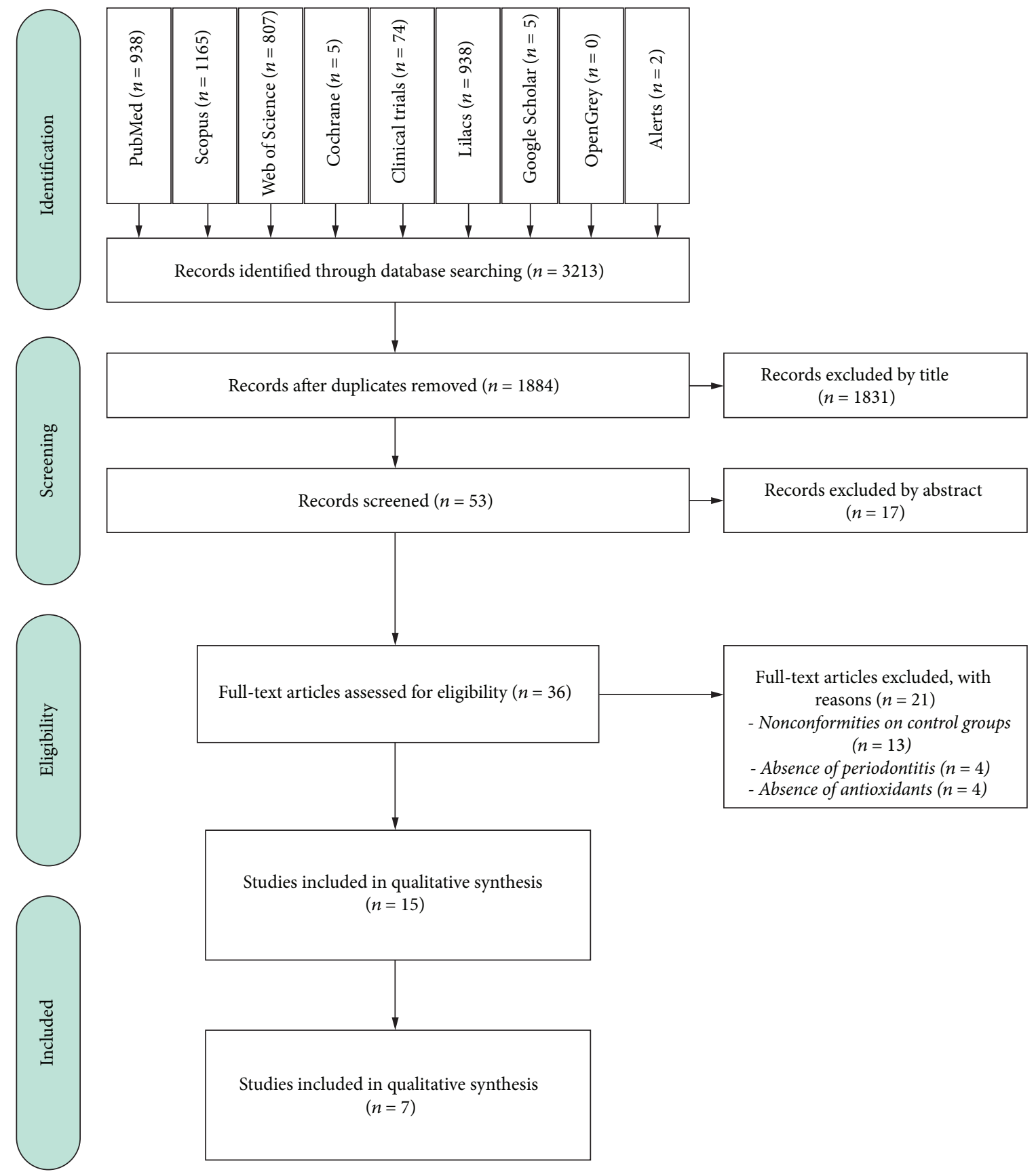

FIgURE 1: Flow diagram of literature search according to the PRISMA statement.

plus antioxidants $(n=68)$ presented a mean CAL, in millimeters, lower than individuals treated with PT only $(n=64)$ (SMD $0.29(0.04,0.55), p=0.03, I^{2}=13 \%, I^{2} p=0.32$; Figure 3).

3.3.2. Meta-Analysis for the Plaque Index (PI). Five clinical trials evaluating the influence of PT additionally to antioxidant therapy in the mean of PI, by analyzed sites, were included in this analysis. Including all studies, the heterogeneity was moderate $\left(I^{2}=47 \%\right)$. Individuals treated with PT plus antioxidants presented a similar mean of PI, per sites, compared to individuals treated with PT only up to one month of follow-up (SMD $0.46(-0.02,0.94), p=0.06$, $\left.I^{2}=74 \%, I^{2} p=0.004\right)$. However, at three months of followup (SMD $\left.0.46(0.24,0.68), p<0.0001, I^{2}=0 \%, I^{2} p=0.95\right)$ and in the pooled results (SMD $0.41(0.18,0.64), p=0.0005$, $\left.I^{2}=47 \%, I^{2} p=0.05\right)$ individuals treated with PT plus antioxidants presented a lower mean of PI, per sites, compared to individuals treated with PT only (Figure 4).

3.3.3. Meta-Analysis for the Gingival Index (GI). Six clinical trials evaluating the influence of PT additionally to antioxidant therapy in the mean of GI, per sites, were included in this analysis. Including all studies, the heterogeneity was 


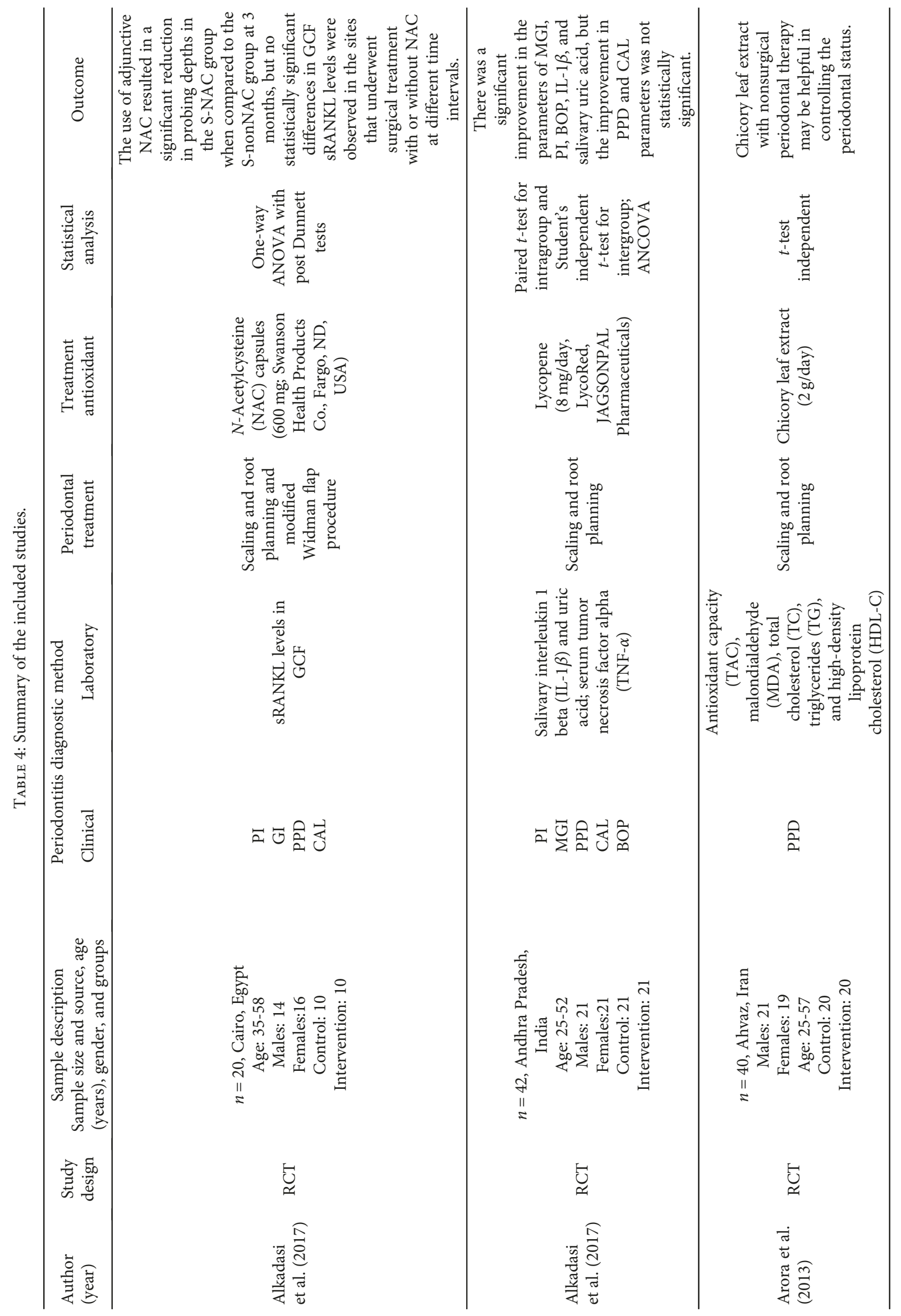




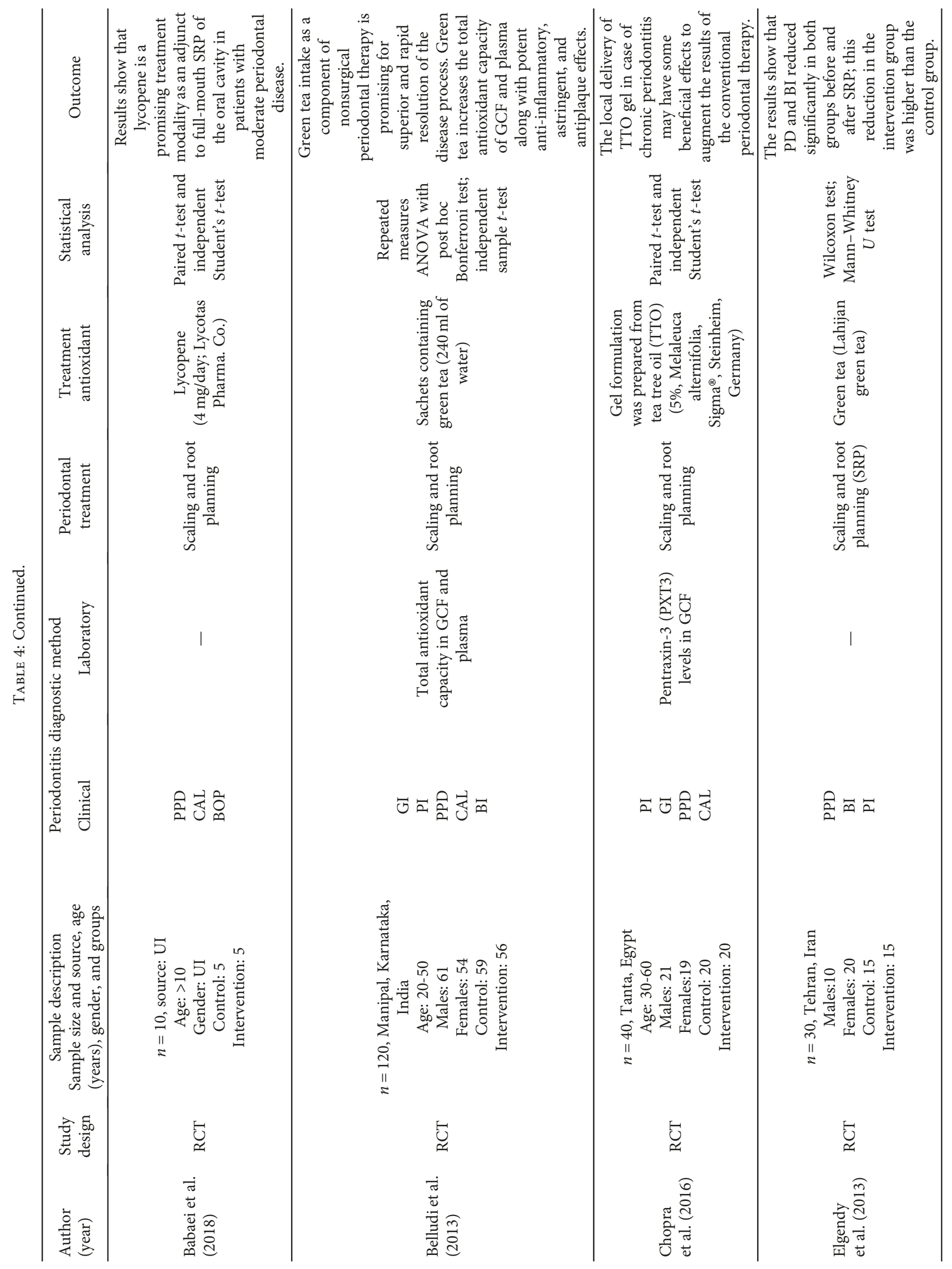




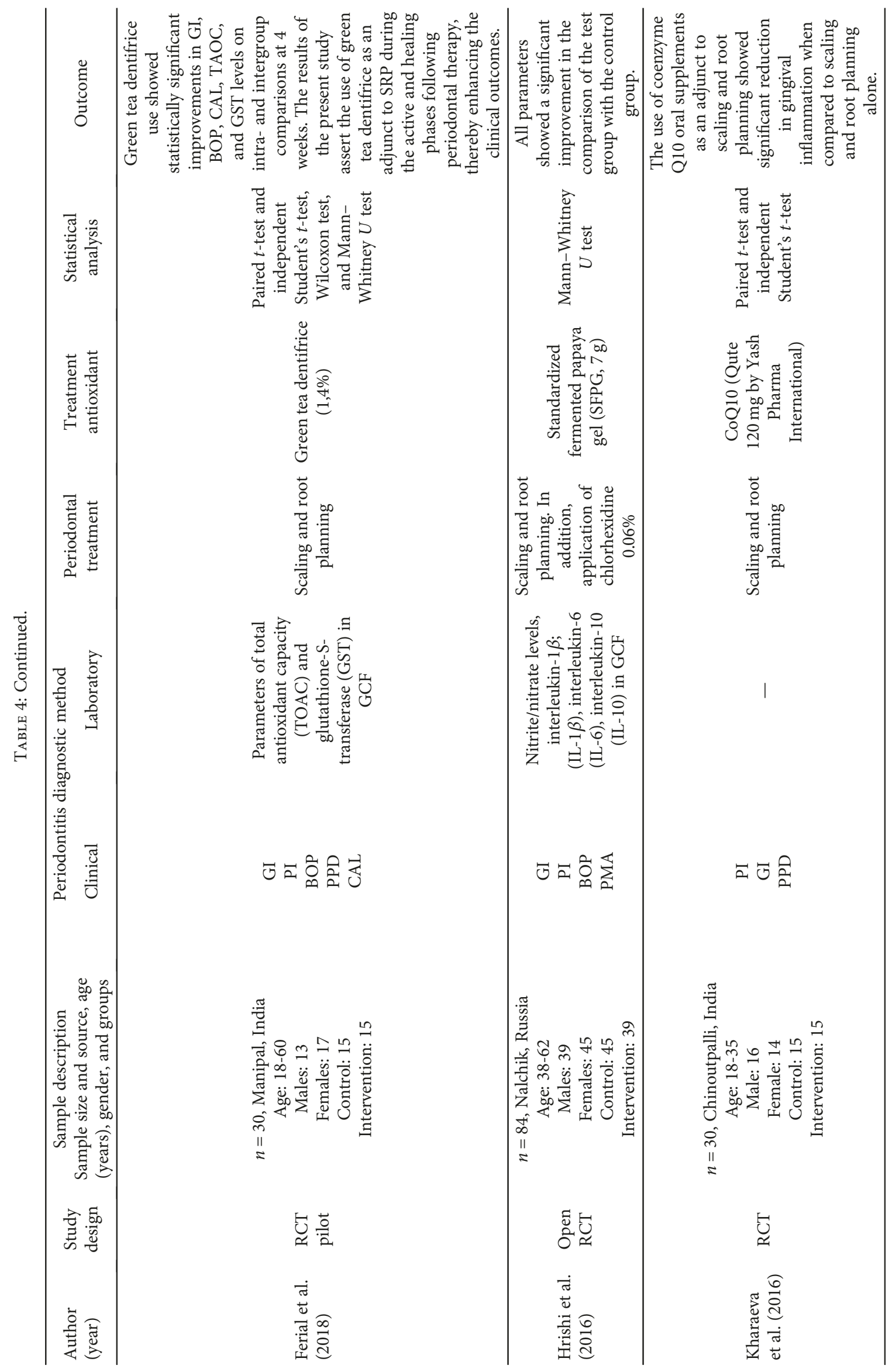




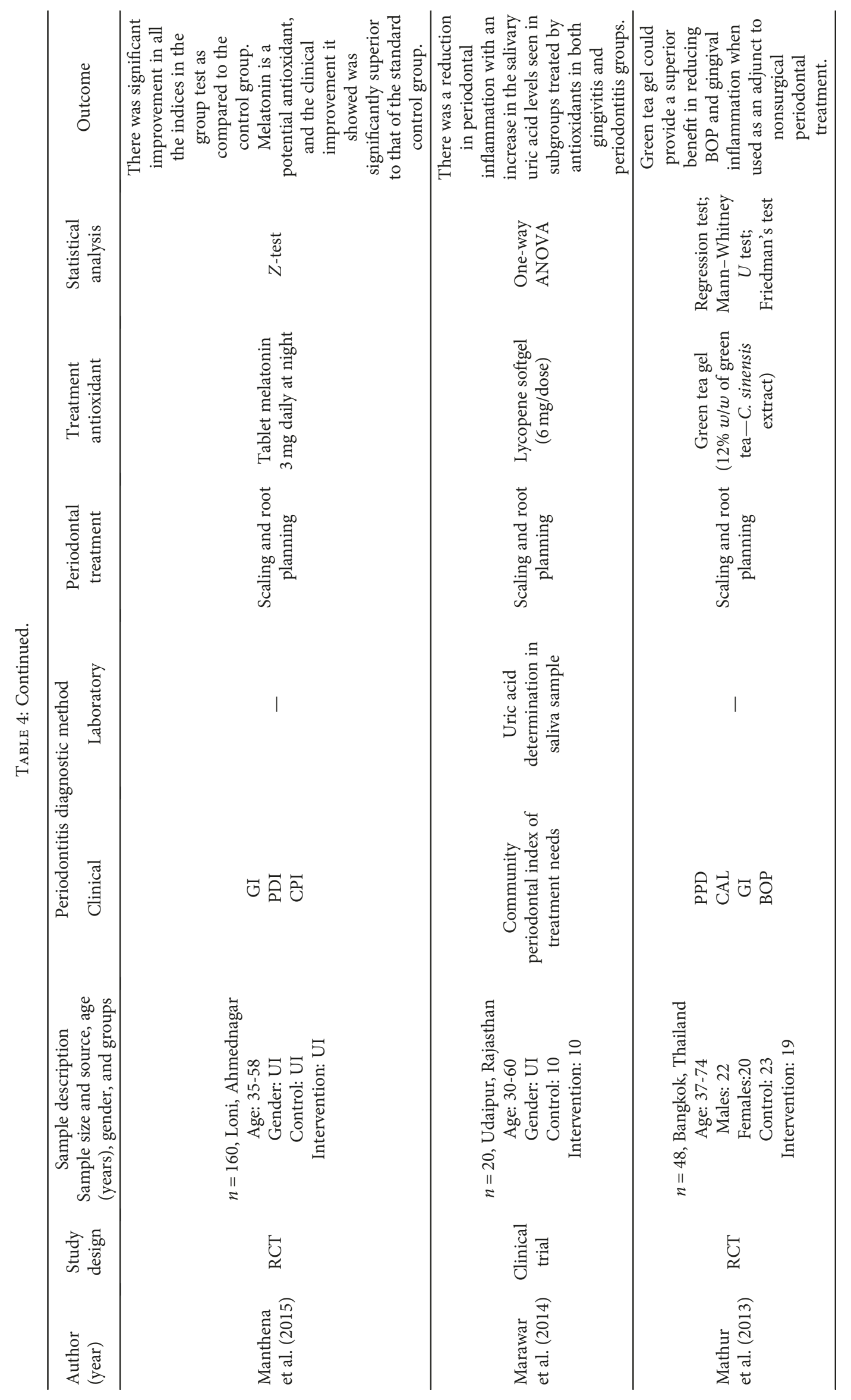




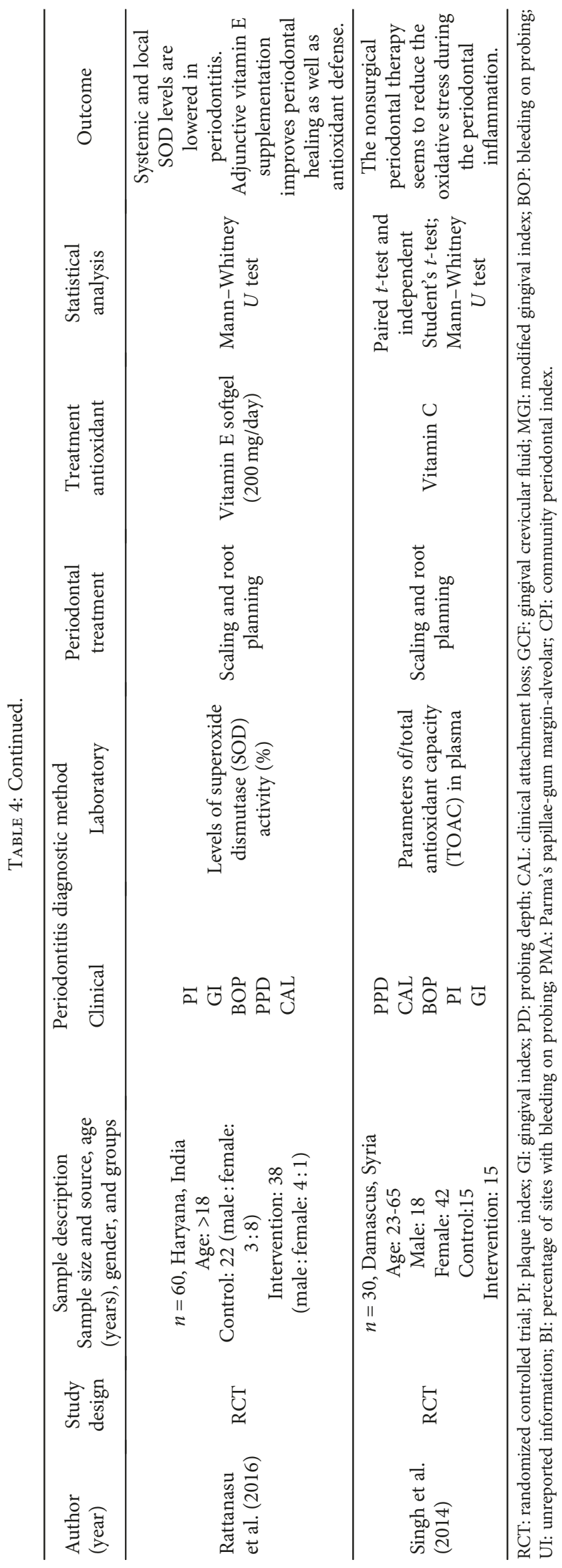




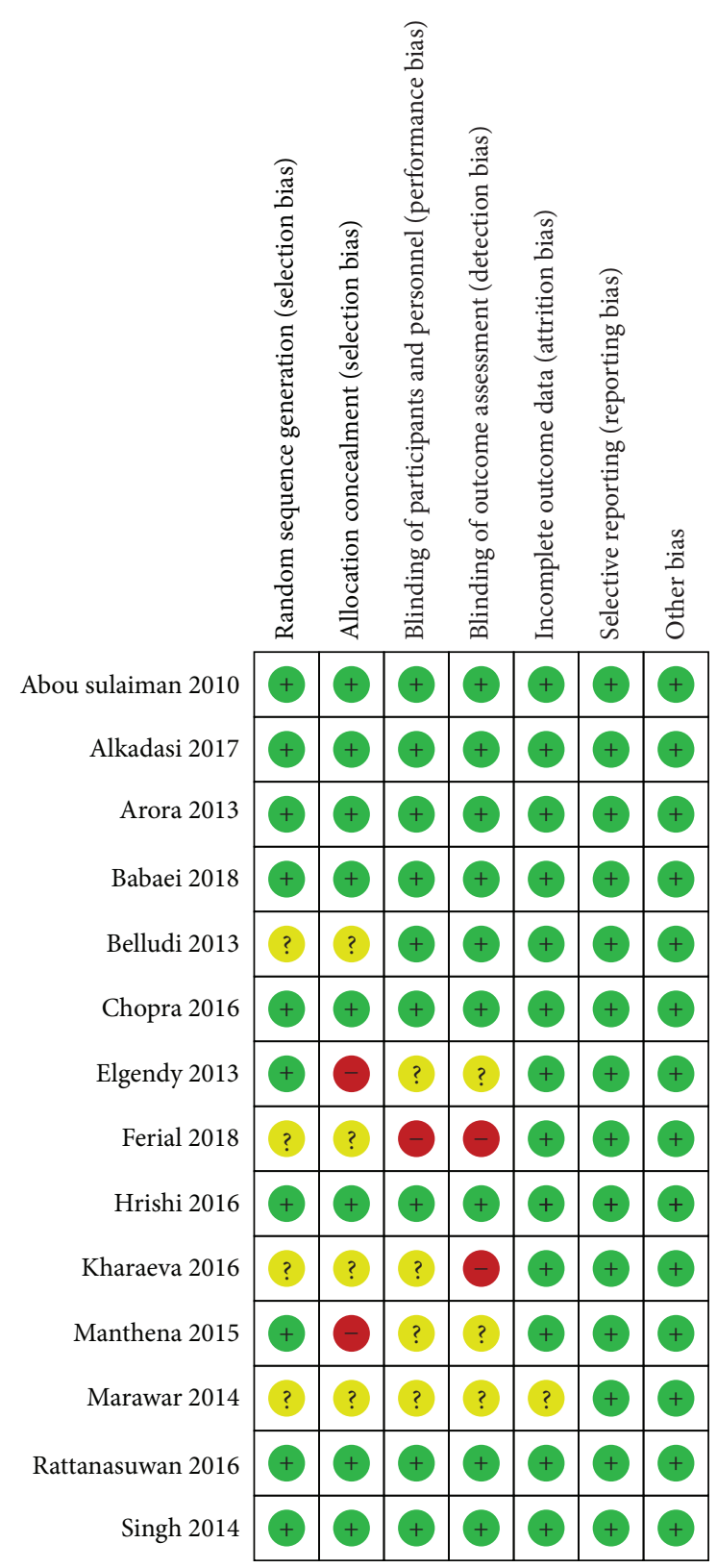

FIGURE 2: Risk of bias evaluation in randomized trials (Cochrane Collaboration's tool).

moderate $\left(I^{2}=43 \%\right)$. At six months or more of follow-up, point individuals treated with PT plus antioxidants presented a similar mean of GI, per sites, compared to individuals treated with PT only (SMD $0.22(-0.43,0.88) p=0.50$, $I^{2}=35 \%, I^{2} p=0.22$ ). Up to one month (SMD 0.43 $\left.(0.23,0.63), p<0.0001, I^{2}=0 \%, I^{2} p=0.76\right)$, up to three months (SMD $0.66(0.30,1.02), p=0.0003, I^{2}=56 \%, I^{2}$ $p=0.06$ ), and in pooled results (SMD $0.51(0.31,0.71)$ $\left.p<0.00001, I^{2}=43 \%, I^{2} p=0.05\right)$, individuals treated with PT plus antioxidants $(n=199)$ presented a lower mean of GI, per sites, compared to individuals treated with PT only $(n=198)$ (Figure 5).
3.3.4. Meta-Analysis of Bleeding on Probing (BOP). Three clinical trials evaluating the influence of $\mathrm{PT}$ plus antioxidants in the mean of BOP per tooth and two per site were included. With the intention of including as many studies as possible, this analysis was conducted with the three studies that evaluate the BOP per tooth and a substantial heterogeneity was observed $\left(I^{2}=64 \%\right)$. During sensitivity analysis, the heterogeneity ranges from $0 \%$ to $73 \%$, and to reduce the overall heterogeneity, the three-month follow-up results of Chopra et al. [23] were excluded from the final analysis. Individuals treated with PT plus antioxidants $(n=86)$ presented a lower mean of BOP, per tooth, compared to individuals treated with PT only $(n=89)$ up to one month (SMD $0.56(0.25$, $\left.0.87), p=0.0004, I^{2}=3 \%, I^{2} p=0.36\right)$ and in pooled results (SMD 0.55 (0.27, 0.83), $p=0.0001, I^{2}=0 \%, I^{2} p=0.54$ ) (Figure 6).

3.3.5. Meta-Analysis Probing Depth (PD). Six clinical trials evaluating the influence of PT plus antioxidants in the mean of PD, in millimeters per tooth, were included. The pooled heterogeneity was considerable $\left(I^{2}=70 \%\right)$. During sensitivity analysis, the heterogeneity ranges from $0 \%$ to $71 \%$, and in an attempt to reduce the overall and subgrouped heterogeneity, the study of Chopra et al. [23] was excluded from the final analysis. Individuals treated with $\mathrm{PT}$ plus antioxidants $(n=68)$ presented a mean of PD similar to individuals treated with PT only $(n=64)$ (SMD $0.13(-0.11,0.36)$, $\left.p=0.3, I^{2}=0 \%, I^{2} p=0.71\right)$, independent of follow-up point evaluated (up to one month (SMD $0.15(-0.19,0.49)$, $p=0.39, I^{2}=0 \%, I^{2} p=0.87$ ), three months (SMD 0.25 $\left.(-0.28,0.79), p=0.36, I^{2}=37 \%, I^{2} p=0.20\right)$, or six months or more (SMD $-0.02(-0.61,0.57), p=0.94, I^{2}=23 \%, I^{2}$ $p=0.25)$ ) (Figure 7).

3.4. Level of Evidence. To assess the quality of evidence across studies, the GRADE approach was applied. Three different evaluations were performed: overall evaluation of the antioxidant effect regarding periodontal indexes (Table 6), the role of lycopene (Table 7), and the role of green tea (Table 8 ) in these outcomes. In the overall analysis, a moderate to high quality was observed among the outcomes, in which the flaws presented in the risk of bias were directly associated with the downgrade of the evidence. In lycopene and green tea (GT) evaluation, a moderate certainty and a high certainty were, respectively, detected.

\section{Discussion}

Fifteen clinical trials studies were included in this systematic review, and all of them indicated the beneficial effects of antioxidants during periodontitis treatment. Our meta-analyses showed improvement in the parameters of clinical attachment loss, plaque index, gingival index, and bleeding on probing (except in probing depth); thus, the results may lead to a possible reduction of periodontal inflammation-a pattern among patients. Considering the limitations found in periodontal treatment for complete resolution of the inflammatory process, it is interesting to seek additional adjuvants to the only mechanical treatment. 


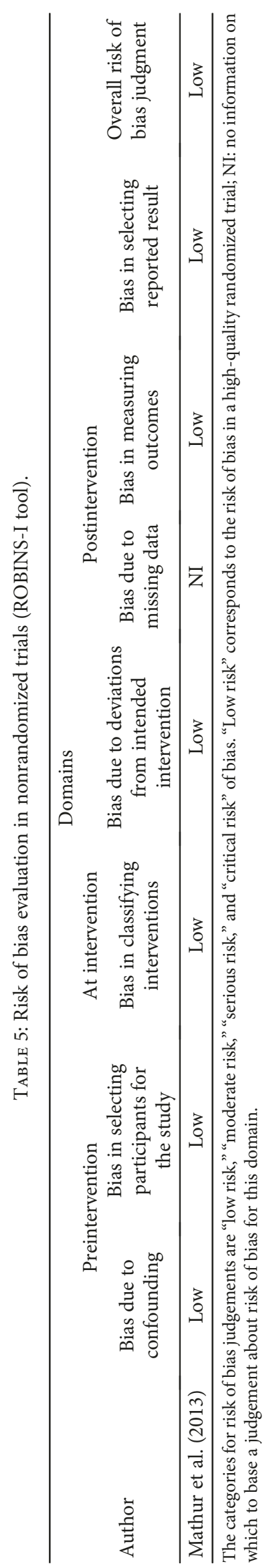




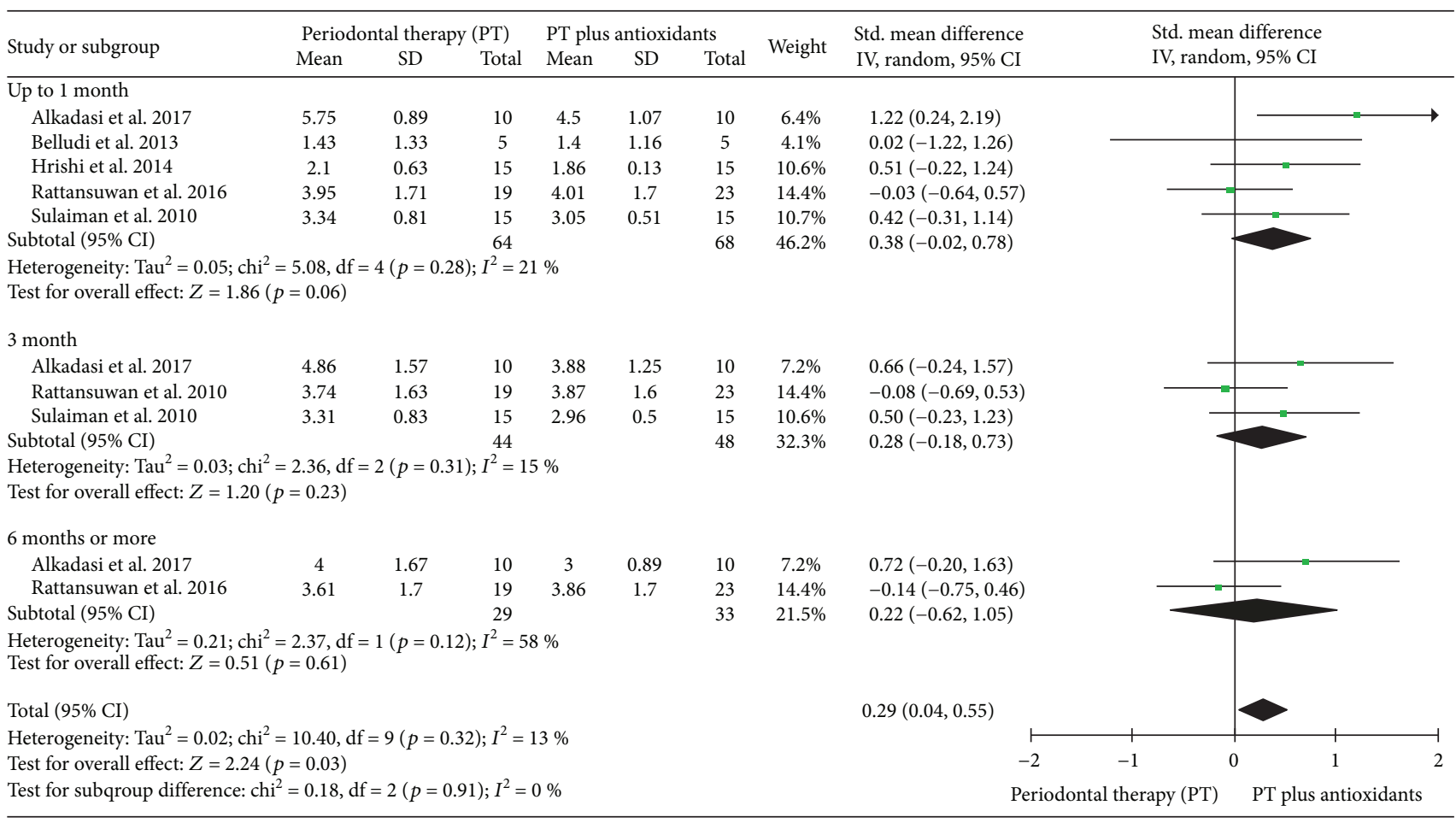

Figure 3: Forest plot of the first meta-analysis for clinical attachment loss (CAL).

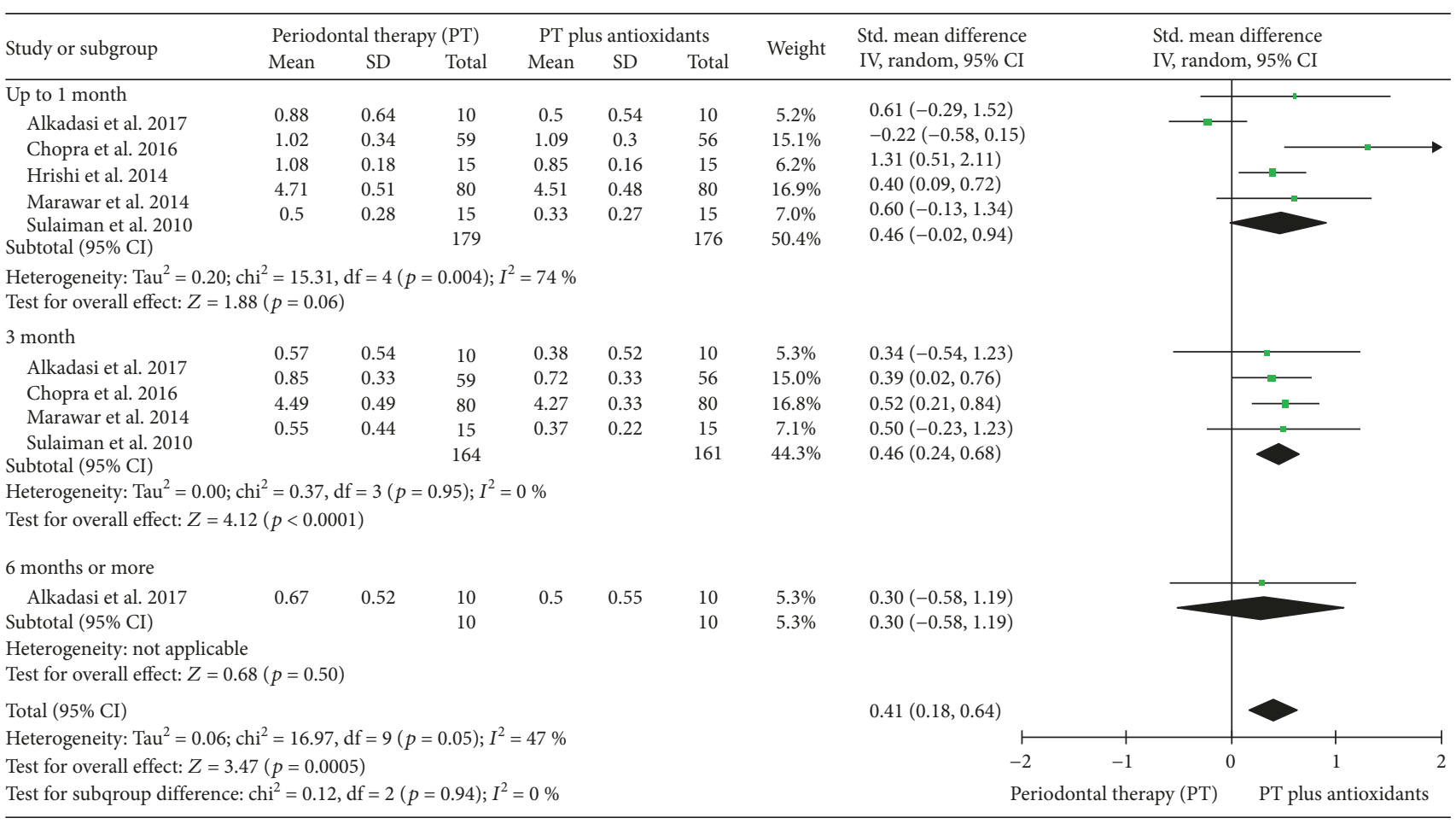

FIGURE 4: Forest plot of the second meta-analysis for the plaque index (PI).

Furthermore, the complexity of interactions between the microbiota, host, and environment must be considered in periodontal therapy. Therefore, additional strategies on treat- ment and self-care has to be investigated. Clinically, the reduction of the inflammatory pattern to low levels is important to control the periodontal health state. It is important to 


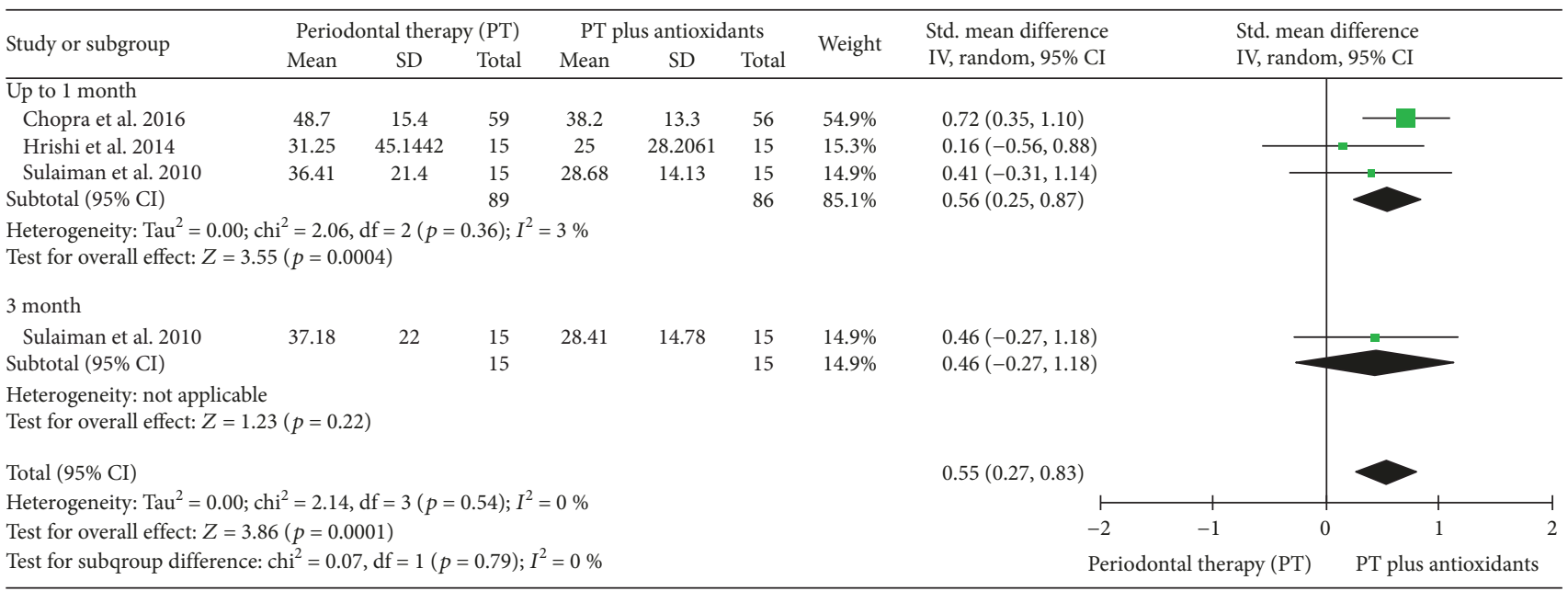

FIGURE 5: Forest plot of the third meta-analysis for the gingival index (GI).

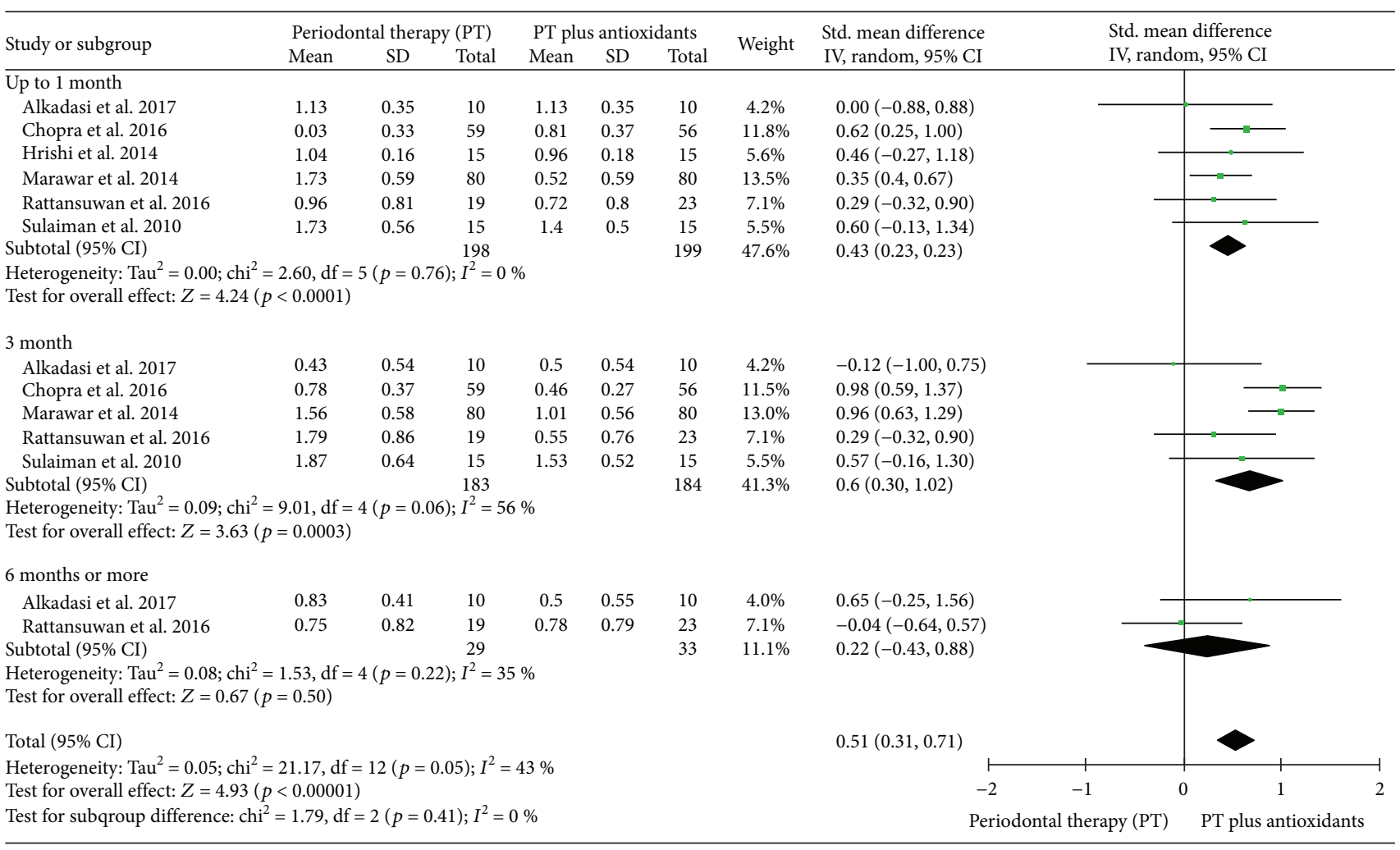

FIgURE 6: Forest plot of the fourth meta-analysis for bleeding on probing (BOP).

mention that the inflammation decrease is essential to establish the health of the periodontal tissues [3]. It is relevant to expose that in patients with severe inflammatory conditions, the reduction of the inflammatory status is essential because it contributes to the planning of periodontal health maintenance or management of reduced periodontium in regenerative therapies $[3,12]$. Therefore, because there is an important relationship between the presence of reactive oxygen species and an inflammatory condition, the antioxidant therapy may control the disease.
In this context, etiopathogenic knowledge is fundamental. There are evidences in the literature that point to the role of oxidative stress with a decrease in the antioxidant defenses that stimulate the process of periodontal destruction [34]. The relationship between oxidative stress and periodontal disease is quite strong and can be a two-way path. On one hand, the presence of periodontal inflammation increases the number of oxidative stress markers, and on the other hand, it tends to potentiate aspects of periodontal destruction [35]. 


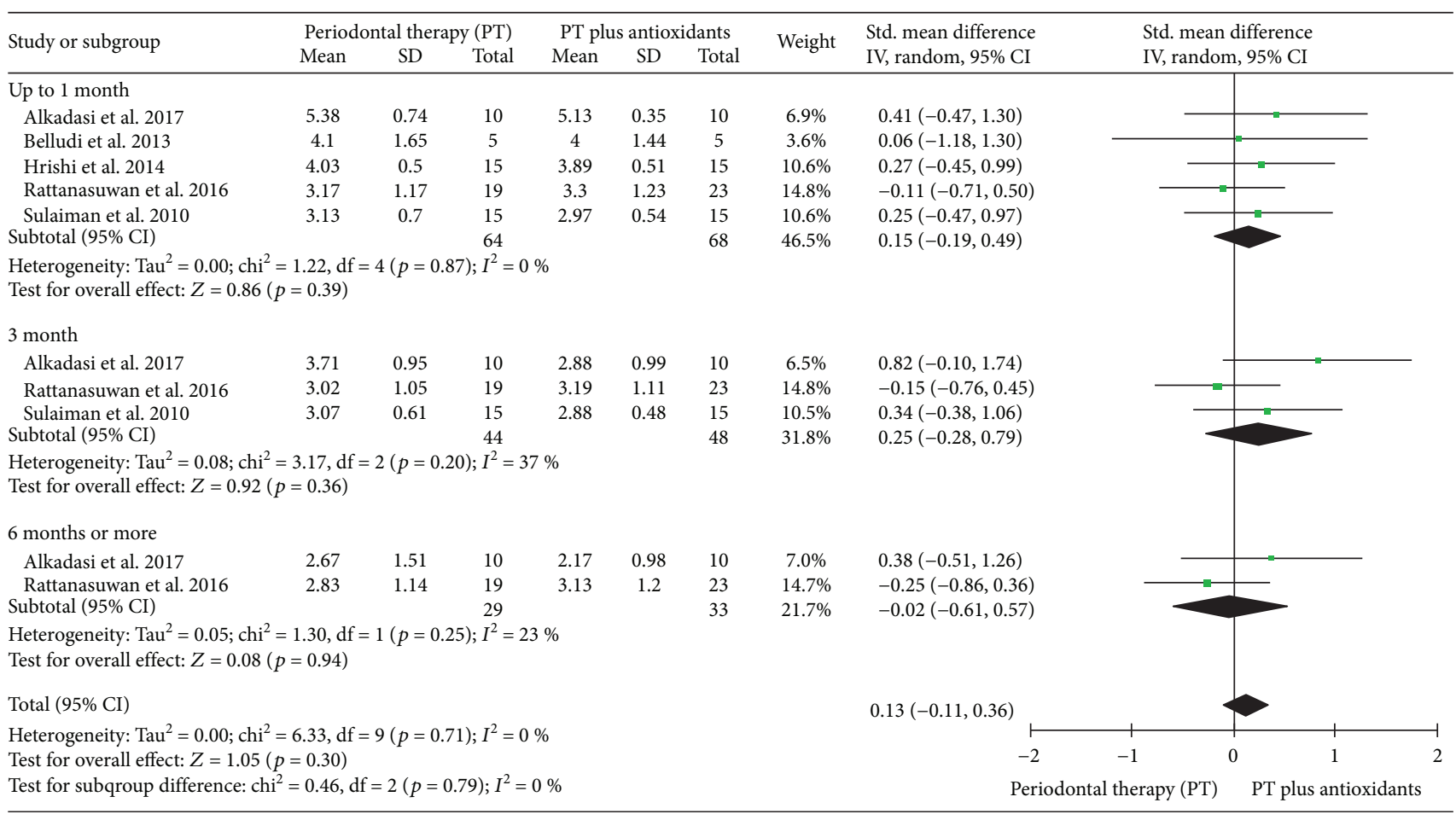

FIgURE 7: Forest plot of the fifth meta-analysis for probing depth (PD).

The literature supports the hypotheses about the association pathway between antioxidant defense and improved periodontitis. On the other hand, this association needs support and the establishment of a causal relationship by evidence-based clinical decisions regarding the use of antioxidants [5]. In this sense, it is essential to make a broad review of the existing literature for a more accurate picture of this relationship. According to the author, there is not any published article about this systematic review and metaanalysis. It was carried out with the contemporary methodological principles trying to reflect the highest degree of available evidence in this approach.

A systematic review involves the application of methodological strategies that limit bias and evaluate and summarize crucial scientific evidences. These systematic analyzes can help practitioners be aware of the scientific literature [36]. The instruments for quality evaluation and biases of these reviews vary according to the type of study. Besides, systematic reviews may include meta-analysis, in which statistical techniques are used to assess the size of the effect of outcomes. In addition, the level of evidence performed using the GRADE tool enables the elaboration of recommendations for clinical practice [18].

The search strategy used in this study included the most important databases to health science in addition to the PICO's strategy, which allows the comparison of the clinical trial results, verifying if there is an additional effect in the use of antioxidants as adjuvants in conventional PT. The search results show that this approach has been slightly studied, especially in considering the antioxidant diversity, as well as the evaluated parameters. Nevertheless, knowing that the antioxidants used have common objectives, the results can be interpreted as a role of antioxidants in general as adjuvants to conventional PT.

A critical point in the context of this review is the type of outcome evaluated, i.e., in which the studies considered periodontal clinical parameters. It can be observed that the PD was the most used parameter. The PD, in the context of the result of periodontal treatment, is one of the most interesting parameters since it is related to the inflammatory status [3]. Changes in PD are related to changes in clinically detectable inflammation [37]. Thus, taking into account that the analysis of the present study is related to the posttreatment outcome, the outcome seems adequate. Obviously, for the longitudinal evaluation of the outcome of periodontal treatment, the measurement of clinical levels of insertion is fundamental.

However, the literature has pointed out that the absence of clinically detectable inflammation is associated with stability of periodontal destruction $[38,39]$. In this sense, the best predictor of periodontal stability is the absence of bleeding on probing $[3,40]$. The presence of inflammation is not a good indicator of destruction, but its absence is related to the stoppage of destruction. Therefore, results related to the PD and different forms of evaluation of inflammation, especially with bleeding on probing, are essential. Analysis of marginal inflammation through the GI or its derivatives does not appear to be a suitable parameter. In this review, the importance given by the authors to the markers of inflammation is clear. The plaque evaluation performed in the studies only seems to have the value to verify the effectiveness of selfcare because there is no support to affirm the effect of 


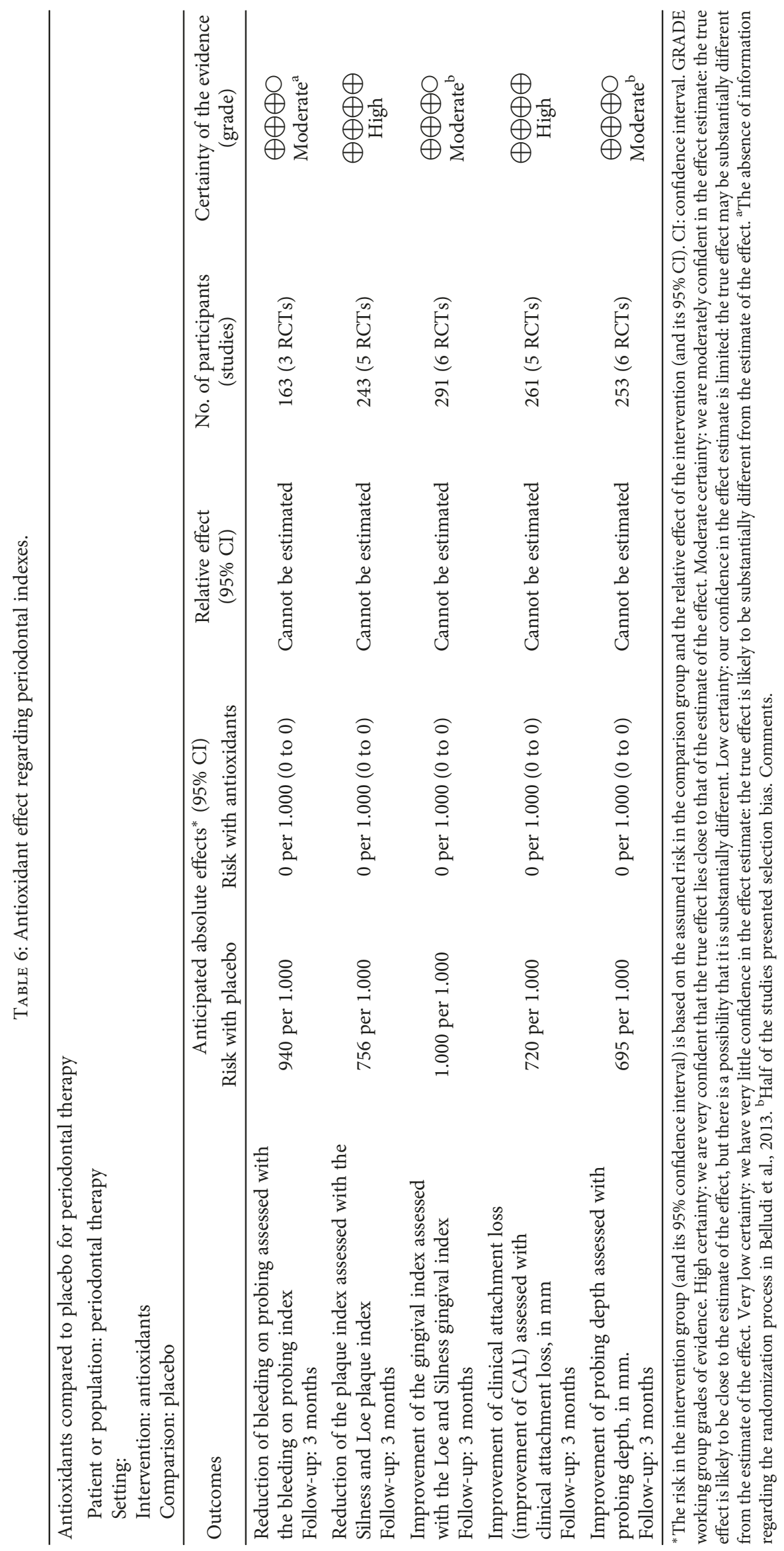




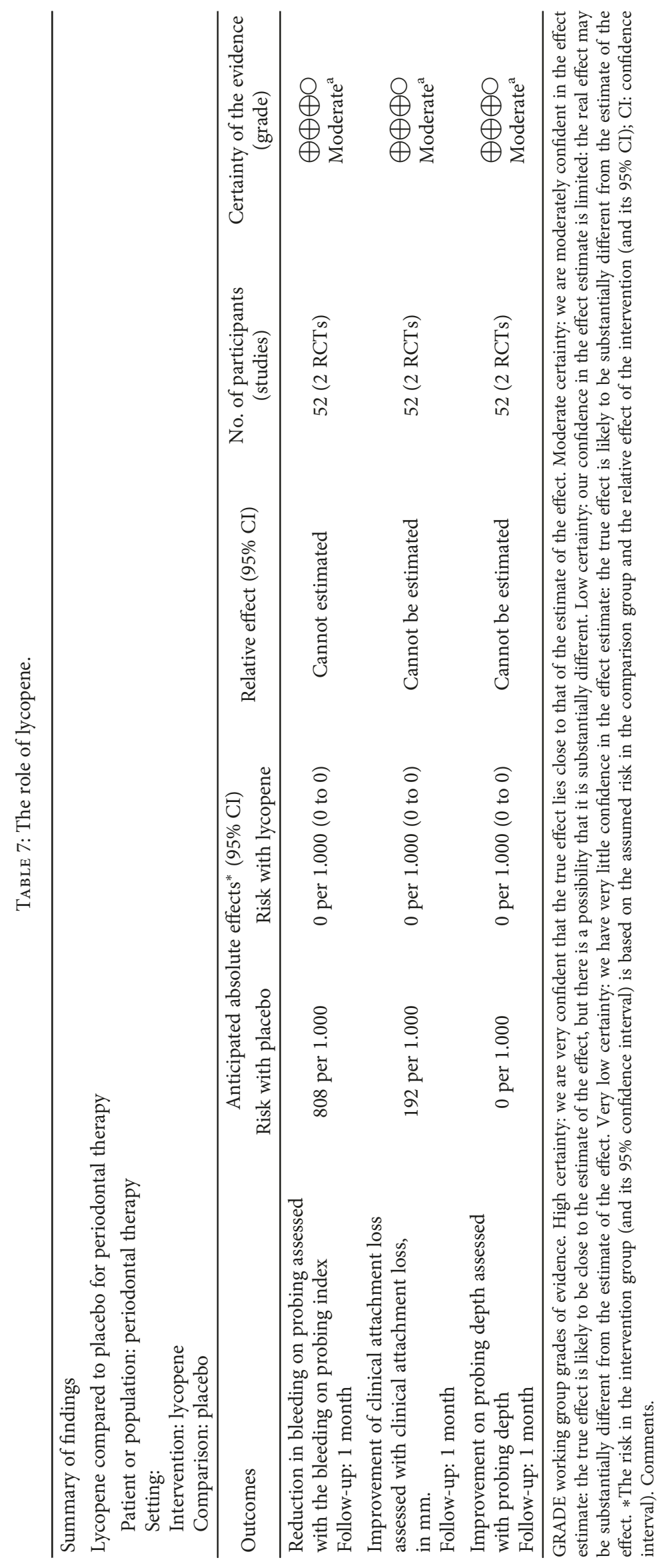




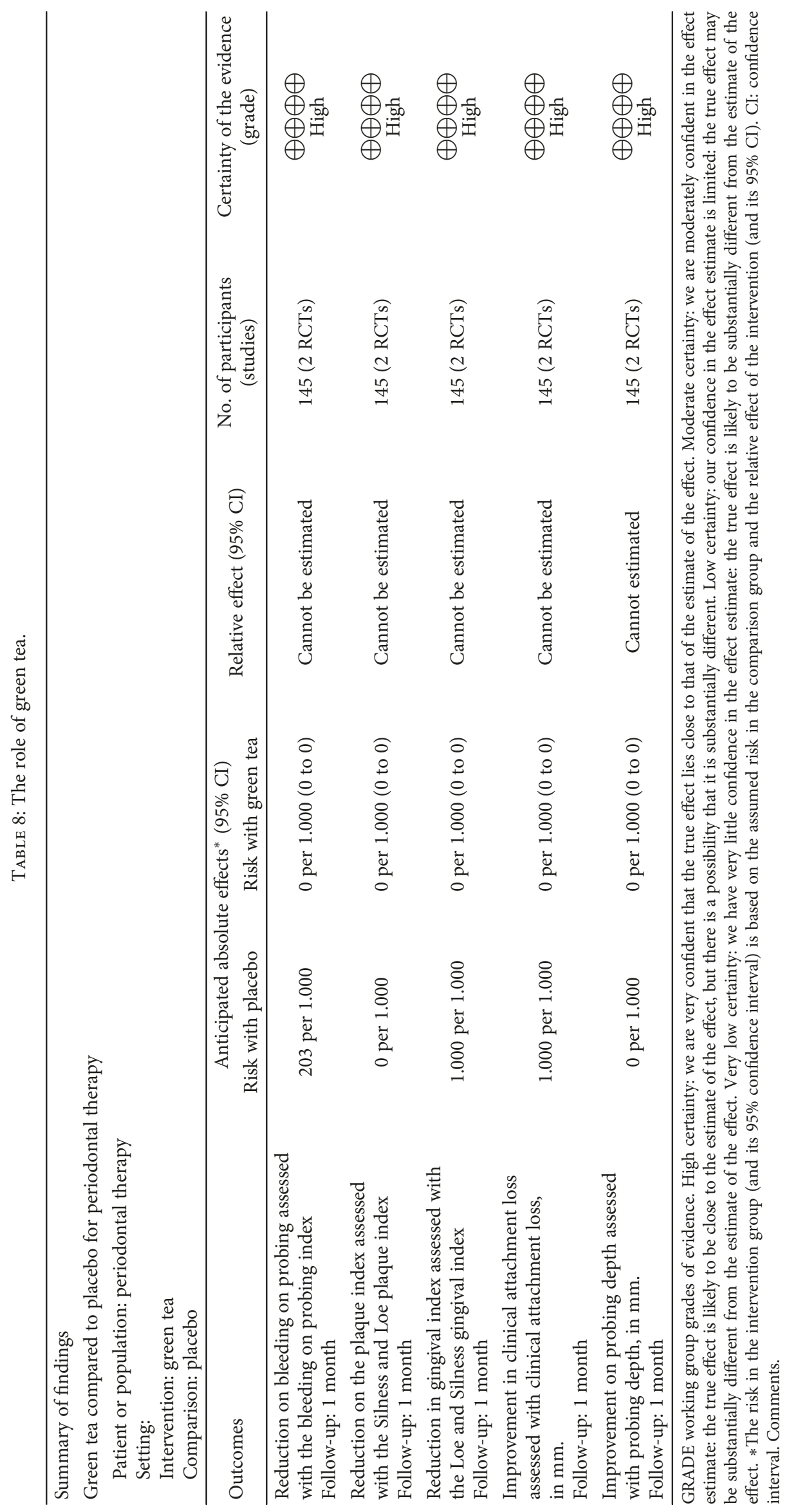


antioxidants on microorganisms of the oral microbiome. Moreover, the patient follow-up through these parameters is important to evaluate the disease course and regression.

Regarding the risk of bias evaluation of the included studies, four studies demonstrated a high risk of bias [24, 26, 27, 33]. Khareava et al. (2016) and Ferial et al. (2018) presented a lack of blinding of both the research participants and the evaluator of the numerical data tabulated between the groups. The blinded experiment in a clinical study helps the reduction of differential assessment of data, changes in interventions applied by the workforce and patients, and the improvement of adherence or retention of study participants, limiting biases during the research [41].

The studies of Elgendy et al. [24] and Manthena et al. [27], besides presenting nonconformities in the blinding, presented a high risk of bias due to the absence of allocation. A detailed description of the method used to generate the random sequence allows to evaluate the possibility of producing comparable groups and reproducing the method in future studies [42].

It is important to emphasize that the quality of the studies presented in this topic is quite impressive, with most studies included presenting a low risk of bias. One of the concerns is the lack of uniformity of the antioxidants used. The number of patients included in the individual studies is quite variable. The use of meta-analyses is aimed at emerging results from different studies, increasing the number of people analyzed, which generates an increase in statistical power.

Considering the problems regarding methodological quality, four studies were excluded from the meta-analysis $[24,26,27,33]$. Our quantitative synthesis grouped the studies according to the following periodontal clinical outcomes: CAL, BOP, PI, GI, and PD. In addition, we subgrouped in each periodontal analysis the follow-up until 1 month, three months, and six months in order to compare the periodontal condition before and after the therapies studied.

The results of the meta-analyses revealed that using antioxidants as adjuvants to PT improved the following clinical parameters: CAL, BOP, and GI, demonstrating $p$ values for the test of significance of the total overall estimate of 0.03 , 0.001 , and $<0.0001$, respectively. These data confirm that this therapy has been able to modulate the periodontal tissues to the point of making them clinically healthy due to the absence or very low levels of inflammation. As for PI, the meta-analysis showed a difference with a $p$ value for the test of significance of the total overall estimate of 0.0005 and especially 3 months after the therapy $(p<0.0001)$. Of the studies included in this meta-analysis, three presented an antioxidant contact with the dental surface. Thus, this contact may stimulate an astringent effect on plaque $[23,25,32]$. Despite the improvement in the inflammation of patients with periodontitis, the meta-analysis for probing depth obtained a value of 0.30 and showed no statistical difference at any follow-up period.

Generally, the results presented point to more significant reductions in inflammatory parameters when antioxidants are used in comparison to placebo. The GRADE tool showed a moderate to high certainty in the quality of evidence depending not only to the clinical parameter but also to the type of antioxidants used. The clinical significance of the difference between these two therapies should be analyzed warily. However, it is important to note that, regardless of quantity, the reduction of inflammation is significant and deserves attention. Apparently, due to the diversity of the agents used, it is essential that a separate analysis be performed for each of the antioxidants used. The most commonly used antioxidants were lycopene and green tea.

About lycopene, the data showed greater efficacy of this carotenoid in relation to the other antioxidants. The possible explanation for such finding is that this compound has high radical scavenging ability and interferes with other nonoxidizing mechanisms, including anti-inflammatory agents, as reported below [43].

Lycopene belongs to the family of carotenoids, it is an open-chain isomer of $\beta$-carotene, one of the primary antioxidants in the diet. A natural pigment synthesized by lycopene is responsible for the red color of many fruits and vegetables, such as ripe tomatoes, watermelons, and papayas [44]. Lycopene may accumulate in the lipophilic compartments of the membrane or lipoprotein, thereby being transported by plasma lipoproteins, and the distribution depends on their chemical structure. As a lipophilic compound, lycopene is mainly transported by low-density lipoproteins and can be found in the adrenal gland, liver, prostate, and reproductive tissues (ten times higher than other tissues) [45, 46]. However, the bioavailability of lycopene was higher in the large intestine $(57 \%)$ than in the small intestine $(40 \%)$ but its absorption potential was insignificant in the large intestine [47]. The biological effects of lycopene are associated with its antioxidant and nonoxidizing actions, such as antiinflammatory and cell signalling activities, which are well established. Its antioxidant activity is related to binding to reactive oxygen species (ROS) by different mechanisms: (i) by electron transfer, (ii) by hydrogen atom transfer, or (iii) by adduct formation [48]. Di Mascio et al. reported that lycopene has singlet oxygen quenching ability, showing that it is two times more effective than $\beta$-carotene, 100 times more potent than $\alpha$-tocopherol, and at least 47 times stronger than vitamin $\mathrm{E}$ [49]. In addition, its antioxidant mechanisms are also associated with the elimination of other free radicals, leading to the reduction of intracellular and extracellular ROS levels, and decrease the formation of MDA in plasma and tissue and increase GSH levels and hepatic GSH-Px, SOD, and CAT activities [50]. Also, lycopene also inhibits $\mathrm{NF}-\kappa \mathrm{B}$ activation, DNA fragmentation, caspase -3 activation, and cytochrome $c$ release [51].

In other ways, it activates the NF-E2 p45-related factor 2 (Nrf2)/HO-1 pathway, by mechanisms of direct interaction of lycopene or its metabolites with the protein cysteine residue Keap 1 that induces the expression and translocation of Nrf2, a regulator of expression of antioxidant genes under both physiological and oxidative stress conditions. In addition, it activates kinases that release and translocate $\mathrm{Nrf} 2$ to the nucleus $[50,52]$. The anti-inflammatory action of lycopene can be attributed mainly to the induction of inflammatory mediators, such as interleukin- $1 \beta$ (IL-1 $\beta$ ), IL-6, IL-8, and tumor necrosis factor- $\alpha$ (TNF- $\alpha)$ [53]. 
In the case of GT, its pharmacological effect is mainly due to polyphenols and flavonoids, notably (-) epicatechin (EC), (-) epigallocatechin (EGC), and the derivatives of gallate, such as (-)-epigallocatechin-3-gallate (EGCG), and (-)-epicatechin-3-gallate (ECG), but it has a low concentration of phenolic acids [54]. Several studies have shown that GT has a dubious effect, both antioxidant and prooxidant activities. Its oxidant action can lead to ROS generation, caspase- 3 and caspase- 9 activation, apoptosis induction, and inhibition of the growth of cancer cells, which is essential in cancer prevention and the oncogenic process. The GT can also inhibit the transcription factor activation, such as NF- $\kappa \mathrm{B}$, activator protein-1 (AP-1), and receptor tyrosine kinase pathways (RTKs). The direct antioxidant activity of GT is related mainly to hydrogen atom transfer, single-electron transfer reactions, and both involving hydroxyl groups, as well as increase in the levels of SOD and catalase that can attenuate lipid peroxidation and protein carbonylation under conditions of oxidative stress $[55,56]$. Besides, GT can improve humoral and cellular immunity and has a potent anti-inflammatory effect, inhibiting the level of TNF- $\alpha$ [57].

Despite the evidence of the benefits of antioxidants as adjuvants in periodontal therapy, the substantial limitation was the inclusion of a small number of studies in our quantitative synthesis, suggesting more randomized clinical trials which measure periodontal clinical parameters. Another limitation was the use of not only different types of antioxidants between the 15 included studies but also different administrations (capsules, extracts, sachets, dentifrices, gel formulation, etc.), because our review cannot point a specific adjuvant to the clinical treatment.

Moreover, our results corroborate the hypothesis suggested in the scientific literature that oxidative stress is involved in the pathogenesis of periodontal disease. In general, this systematic review indicates that antioxidants are proper adjuvants to periodontal treatment and can improve the oxidative damage promoted to the periodontal tissue during periodontitis.

\section{Conclusions}

Based on clinical trials, this systematic review suggested the use of antioxidants, especially lycopene and GT, as good adjuvants in periodontal therapy, modulating oxidative stress on the periodontium during periodontitis. Therefore, antioxidant therapy may lead to the maintenance of periodontal heath and decrease of inflammatory levels, such as improvement of PI, GI, BOP, and CAL. Further longitudinal studies to better understand the mechanisms of inflammation decrease are highly recommended.

\section{Conflicts of Interest}

The authors declare that there are no conflicts of interest in this paper.

\section{Authors' Contributions}

MMLC and NND performed the searches, quality assessment, analysis of results, and manuscript elaboration. MMLC, PCN, and NCFF performed data extraction, analysis of results, and manuscript elaboration. MBM and LCM performed quantitative analysis and manuscript elaboration. NCFF and CFM performed the quality checking of evidence and manuscript elaboration. MCM, CKR, and RRL performed analysis of results and manuscript elaboration. Micaele Maria Lopes Castro and Nathallia Neves Duarte are dividing the first authorship.

\section{Acknowledgments}

The authors would like to thank the Federal University of Pará, Federal University of Rio de Janeiro, Federal University of Rio Grande do Sul, and University of Alberta for the technical and scientific support. This work was supported by Pró-Reitoria de Pesquisa e Pós-Graduação da UFPA (PROPESP, UFPA, Brazil). This research was financed in part by the Coordenação de Aperfeiçoamento de Pessoal de Nível Superior, Brasil (CAPES), Finance code 001.

\section{References}

[1] P. N. Papapanou, M. Sanz, N. Buduneli et al., "Periodontitis: consensus report of workgroup 2 of the 2017 World Workshop on the Classification of Periodontal and Peri-Implant Diseases and Conditions," Journal of Periodontology, vol. 89, Suppl 1, pp. S173-s182, 2018.

[2] J. G. Caton, G. Armitage, T. Berglundh et al., "A new classification scheme for periodontal and peri-implant diseases and conditions - introduction and key changes from the 1999 classification," Journal of Clinical Periodontology, vol. 45, pp. S1-s8, 2018.

[3] N. P. Lang and P. M. Bartold, "Periodontal health," Journal of Clinical Periodontology, vol. 45, pp. S9-S16, 2018.

[4] S. Murakami, B. L. Mealey, A. Mariotti, and I. L. C. Chapple, "Dental plaque-induced gingival conditions," Journal of Periodontology, vol. 89, pp. S17-S27, 2018.

[5] L. Tóthová and P. Celec, "Oxidative stress and antioxidants in the diagnosis and therapy of periodontitis," Frontiers in Physiology, vol. 8, 2017.

[6] B. Halliwell and M. Whiteman, "Measuring reactive species and oxidative damage in vivo and in cell culture: how should you do it and what do the results mean?," British Journal of Pharmacology, vol. 142, no. 2, pp. 231-255, 2004.

[7] K. B. F. Barbosa, N. M. B. Costa, R. . C. G. Alfenas, S. O. de Paula, V. P. R. Minim, and J. Bressan, "Estresse oxidativo: conceito, implicações e fatores modulatórios," Revista de Nutrição, vol. 23, no. 4, pp. 629-643, 2010.

[8] I. L. C. Chapple, "Reactive oxygen species and antioxidants in inflammatory diseases," Journal of Clinical Periodontology, vol. 24, no. 5, pp. 287-296, 1997.

[9] J. A. Leopold, "Antioxidants and coronary artery disease: from pathophysiology to preventive therapy," Coronary Artery Disease, vol. 26, no. 2, pp. 176-183, 2015.

[10] F. J. Kelly, "Vitamins and respiratory disease: antioxidant micronutrients in pulmonary health and disease," The Proceedings of the Nutrition Society, vol. 64, no. 4, pp. 510-526, 2005. 
[11] D. Fusco, G. Colloca, M. R. Lo Monaco, and M. Cesari, "Effects of antioxidant supplementation on the aging process," Clinical Interventions in Aging, vol. 2, no. 3, pp. 377-387, 2007.

[12] S. Trivedi and N. Lal, "Antioxidant enzymes in periodontitis," Journal of Oral Biology and Craniofacial Research, vol. 7, no. 1, pp. 54-57, 2017.

[13] D. Moher, A. Liberati, J. Tetzlaff, D. G. Altman, and The PRISMA Group, "Preferred reporting items for systematic reviews and meta-analyses: the PRISMA statement," PLoS Med, vol. 6, no. 7, p. e1000097, 2009.

[14] J. P. T. Higgins, D. G. Altman, P. C. Gotzsche et al., "The Cochrane Collaboration's tool for assessing risk of bias in randomised trials," BMJ, vol. 343, no. oct18 2, p. d5928, 2011.

[15] J. A. Sterne, M. A. Hernán, B. C. Reeves et al., "ROBINS-I: a tool for assessing risk of bias in non-randomised studies of interventions," BMJ, vol. 355, 2016.

[16] J. P. T. Higgins and S. Green, "Cochrane handbook for systematic reviews of interventions version 5.1.0 [updated March 2011]," The Cochrane Collaboration, 2011, http://handbook. cochrane.org.

[17] M. Borenstein, L. V. Hedges, J. P. T. Higgins, and H. R. Rothstein, Introduction to Meta-Analysis, Wiley, 2011.

[18] H. Balshem, M. Helfand, H. J. Schunemann et al., "GRADE guidelines: 3. Rating the quality of evidence," Journal of Clinical Epidemiology, vol. 64, no. 4, pp. 401-406, 2011.

[19] B. Alkadasi, S. Abdulrab, S. Gaafer et al., "Effect of adjunctive use of systemic antioxidant therapy ( $N$-acetylcysteine) on soluble receptor activator nuclear factor $\kappa \mathrm{B}$ ligand levels in gingival crevicular fluid following surgical periodontal treatment for chronic periodontitis," Journal of Oral Science, vol. 59, no. 4, pp. 519-526, 2017.

[20] N. Arora, H. Avula, and J. K. Avula, "The adjunctive use of systemic antioxidant therapy (lycopene) in nonsurgical treatment of chronic periodontitis: a short-term evaluation," Quintessence International, vol. 44, no. 6, pp. 395-405, 2013.

[21] H. Babaei, F. Forouzandeh, L. Maghsoumi-Norouzabad, H. A. Yousefimanesh, M. Ravanbakhsh, and A. Zare Javid, "Effects of chicory leaf extract on serum oxidative stress markers, lipid profile and periodontal status in patients with chronic periodontitis," Journal of the American College of Nutrition, vol. 37, no. 6, pp. 479-486, 2018.

[22] S. A. Belludi, S. Verma, R. Banthia et al., "Effect of lycopene in the treatment of periodontal disease: a clinical study," The Journal of Contemporary Dental Practice, vol. 14, no. 6, pp. 1054-1059, 2013.

[23] A. Chopra, B. S. Thomas, K. Sivaraman, H. K. Prasad, and S. U. Kamath, "Green tea intake as an adjunct to mechanical periodontal therapy for the management of mild to moderate chronic periodontitis: a randomized controlled clinical trial," Oral Health \& Preventive Dentistry, vol. 14, no. 4, pp. 293-303, 2016.

[24] E. A. Elgendy, D. H. Zineldeen, and S. A. M. Ali, "Effect of local application of tea tree (Melaleuca alternifolia) oil gel on long pentraxin level used as an adjunctive treatment of chronic periodontitis: a randomized controlled clinical study," Journal of Indian Society of Periodontology, vol. 17, no. 4, pp. 444-448, 2013.

[25] T. S. Hrishi, P. P. Kundapur, A. Naha, B. S. Thomas, S. Kamath, and G. S. Bhat, "Effect of adjunctive use of green tea dentifrice in periodontitis patients - a randomized controlled pilot study," International Journal of Dental Hygiene, vol. 14, no. 3, pp. 178-183, 2016.

[26] Z. F. Kharaeva, L. R. Zhanimova, M. S. Mustafaev et al., "Effects of standardised fermented papaya gel on clinical symptoms, inflammatory cytokines, and nitric oxide metabolites in patients with chronic periodontitis: an open randomised clinical study," Mediators of Inflammation, vol. 2016, Article ID 9379840, 12 pages, 2016.

[27] S. Manthena, M. V. Rao, L. P. Penubolu, M. Putcha, and A. V. Harsha, "Effectiveness of CoQ10 oral supplements as an adjunct to scaling and root planing in improving periodontal health," Journal of Clinical and Diagnostic Research, vol. 9, no. 8, pp. ZC26-8, 2015.

[28] A. P. Marawar, P. P. Marawar, D. H. Nandal, A. V. Tilak, R. B. Bhalsinge, and A. A. Barde, "Therapeutic Potential of Melatonin in Periodontitis: A Randomised, Placebo Controlled, Double Blind Study," Research Journal of Pharmaceutical, Biological and Chemical Sciences, vol. 5, no. 4, 2014.

[29] A. Mathur, L. Mathur, B. Manohar et al., "Antioxidant therapy as monotherapy or as an adjunct to treatment of periodontal diseases," Journal of Indian Society of Periodontology, vol. 17, no. 1, pp. 21-24, 2013.

[30] K. Rattanasuwan, S. Rassameemasmaung, V. Sangalungkarn, and C. Komoltri, "Clinical effect of locally delivered gel containing green tea extract as an adjunct to non-surgical periodontal treatment," Odontology, vol. 104, no. 1, pp. 89-97, 2016.

[31] N. Singh, S. Chander Narula, R. Kumar Sharma, S. Tewari, and P. Kumar Sehgal, "Vitamin E supplementation, superoxide dismutase status, and outcome of scaling and root planing in patients with chronic periodontitis: a randomized clinical trial," Journal of Periodontology, vol. 85, no. 2, pp. 242-249, 2014.

[32] A. E. Abou Sulaiman and R. M. H. Shehadeh, "Assessment of total antioxidant capacity and the use of vitamin $\mathrm{C}$ in the treatment of non-smokers with chronic periodontitis," Journal of Periodontology, vol. 81, no. 11, pp. 1547-1554, 2010.

[33] F. Taleghani, G. Rezvani, M. Birjandi, and M. Valizadeh, "Impact of Green Tea Intake on Clinical Improvement in Chronic Periodontitis: A Randomized Clinical Trial," Journal of Stomatology, Oral and Maxillofacial Surgery, vol. 119, no. 5, pp. 365-368, 2018.

[34] Y. Wang, O. Andrukhov, and X. Rausch-Fan, "Oxidative stress and antioxidant system in periodontitis," Frontiers in Physiology, vol. 8, p. 910, 2017.

[35] J. C. da Silva, F. W. M. G. Muniz, H. J. R. Oballe, M. Andrades, C. K. Rösing, and J. Cavagni, "The effect of periodontal therapy on oxidative stress biomarkers: a systematic review," Journal of Clinical Periodontology, vol. 45, no. 10, pp. 1222-1237, 2018.

[36] D. J. Cook, C. D. Mulrow, and R. B. Haynes, "Systematic reviews: synthesis of best evidence for clinical decisions," Annals of Internal Medicine, vol. 126, no. 5, p. 376, 1997.

[37] J. Lindhe, S. S. Socransky, S. Nyman, A. Haffajee, and E. Westfelt, "'Critical probing depths" in periodontal therapy," Journal of Clinical Periodontology, vol. 9, no. 4, pp. 323-336, 1982.

[38] R. C. Page and K. S. Kornman, "The pathogenesis of human periodontitis: an introduction," Periodontol 2000, vol. 14, no. 1, pp. 9-11, 1997.

[39] L. Checchi, M. Montevecchi, V. Checchi, and F. Zappulla, "The relationship between bleeding on probing and 
subgingival deposits. An endoscopical evaluation," The Open Dentistry Journal, vol. 3, no. 1, pp. 154-160, 2009.

[40] G. Matuliene, B. E. Pjetursson, G. E. Salvi et al., "Influence of residual pockets on progression of periodontitis and tooth loss: results after 11 years of maintenance," Journal of Clinical Periodontology, vol. 35, no. 8, pp. 685-695, 2008.

[41] K. F. Schulz and D. A. Grimes, "Blinding in randomised trials: hiding who got what," Lancet, vol. 359, no. 9307, pp. 696-700, 2002.

[42] K. Benson and A. J. Hartz, "A comparison of observational studies and randomized, controlled trials," The New England Journal of Medicine, vol. 342, no. 25, pp. 1878-1886, 2000.

[43] J. Shi and M. Le Maguer, "Lycopene in tomatoes: chemical and physical properties affected by food processing," Critical Reviews in Biotechnology, vol. 20, no. 4, pp. 293-334, 2000.

[44] B. L. Lindshield, K. Canene-Adams, and J. W. Erdman Jr., "Lycopenoids: are lycopene metabolites bioactive?," Archives of Biochemistry and Biophysics, vol. 458, no. 2, pp. 136-140, 2007.

[45] S. Agarwal and A. V. Rao, "Tomato lycopene and its role in human health and chronic diseases," CMAJ, vol. 163, no. 6, pp. 739-744, 2000.

[46] K. W. Kong, H. E. Khoo, K. N. Prasad, A. Ismail, C. P. Tan, and N. F. Rajab, "Revealing the power of the natural red pigment lycopene," Molecules, vol. 15, no. 2, pp. 959-987, 2010.

[47] I. Goni, J. Serrano, and F. Saura-Calixto, "Bioaccessibility of beta-carotene, lutein, and lycopene from fruits and vegetables," Journal of Agricultural and Food Chemistry, vol. 54, no. 15, pp. 5382-5387, 2006.

[48] N. I. Krinsky and K. J. Yeum, "Carotenoid-radical interactions," Biochemical and Biophysical Research Communications, vol. 305, no. 3, pp. 754-760, 2003.

[49] P. Di Mascio, S. Kaiser, and H. Sies, "Lycopene as the most efficient biological carotenoid singlet oxygen quencher," Archives of Biochemistry and Biophysics, vol. 274, no. 2, pp. 532-538, 1989.

[50] K. Sahin, C. Orhan, H. Yazlak, M. Tuzcu, and N. Sahin, "Lycopene improves activation of antioxidant system and Nrf2/HO1 pathway of muscle in rainbow trout (Oncorhynchus mykiss) with different stocking densities," Aquaculture, vol. 430, pp. 133-138, 2014.

[51] S. Lim, S. Hwang, J. H. Yu, J. W. Lim, and H. Kim, "Lycopene inhibits regulator of calcineurin 1-mediated apoptosis by reducing oxidative stress and down-regulating Nucling in neuronal cells," Mol Nutr Food Res, vol. 61, no. 5, 2017.

[52] K. Sahin, H. Yazlak, C. Orhan, M. Tuzcu, F. Akdemir, and N. Sahin, "The effect of lycopene on antioxidant status in rainbow trout (Oncorhynchus mykiss) reared under high stocking density," Aquaculture, vol. 418-419, pp. 132-138, 2014.

[53] L. N. Jiang, Y. B. Liu, and B. H. Li, "Lycopene exerts antiinflammatory effect to inhibit prostate cancer progression," Asian Journal of Andrology, vol. 21, no. 1, p. 80, 2019.

[54] M. C. Monteiro and I. Peluso, "The influence of phenolic compounds from coffee and tea on postprandial cardiovascular stress: a mini-review," Current Opinion in Food Science, vol. 13, pp. 63-72, 2017.

[55] S. A. Khan, S. Priyamvada, W. Khan, S. Khan, N. Farooq, and A. N. K. Yusufi, "Studies on the protective effect of green tea against cisplatin induced nephrotoxicity," Pharmacological Research, vol. 60, no. 5, pp. 382-391, 2009.
[56] J. D. Lambert and R. J. Elias, “The antioxidant and pro-oxidant activities of green tea polyphenols: a role in cancer prevention," Archives of Biochemistry and Biophysics, vol. 501, no. 1, pp. 65-72, 2010.

[57] Y. Shirakami and M. Shimizu, "Possible Mechanisms of Green Tea and Its Constituents against Cancer," Molecules, vol. 23, no. 9 , p. 2284, 2018. 


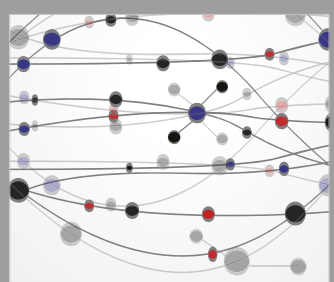

The Scientific World Journal
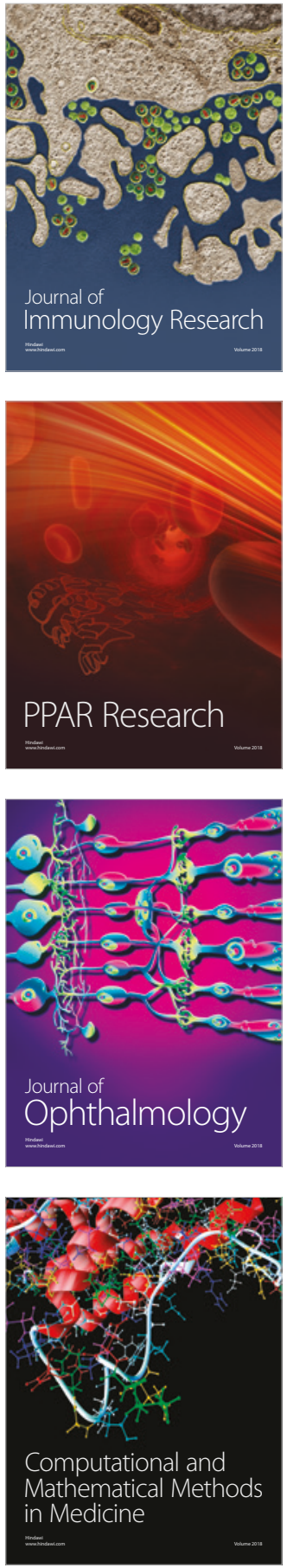

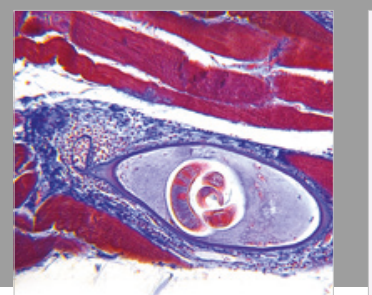

Gastroenterology Research and Practice

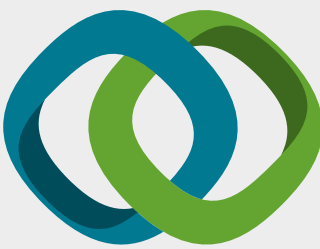

\section{Hindawi}

Submit your manuscripts at

www.hindawi.com
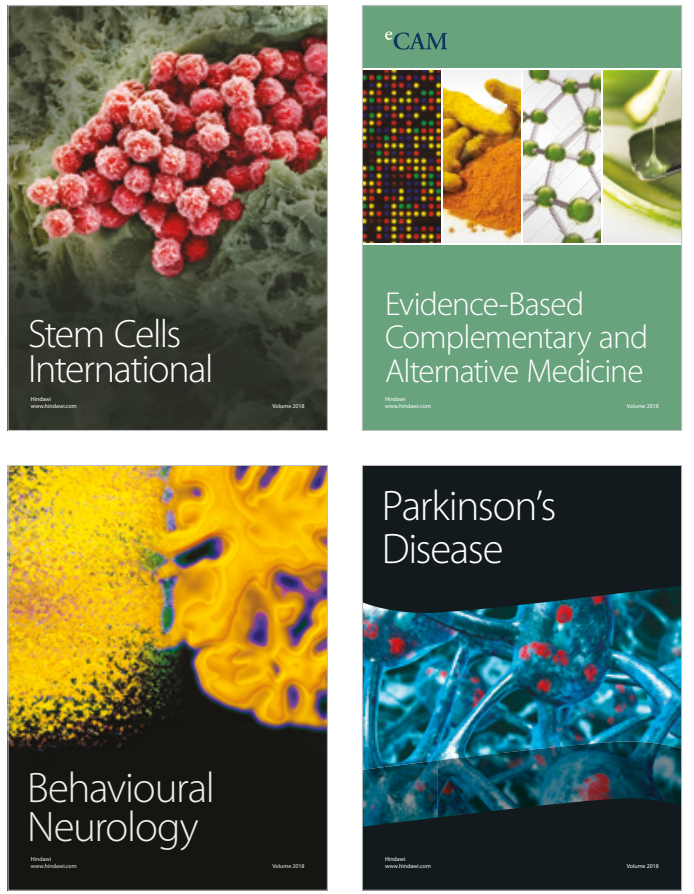

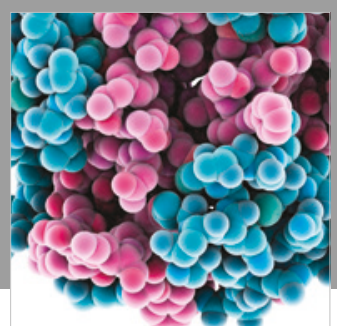

ournal of

Diabetes Research

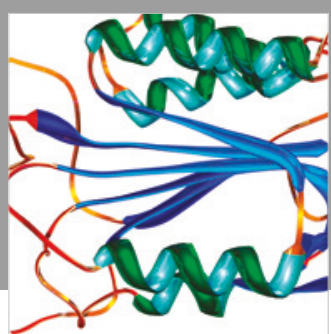

Disease Markers
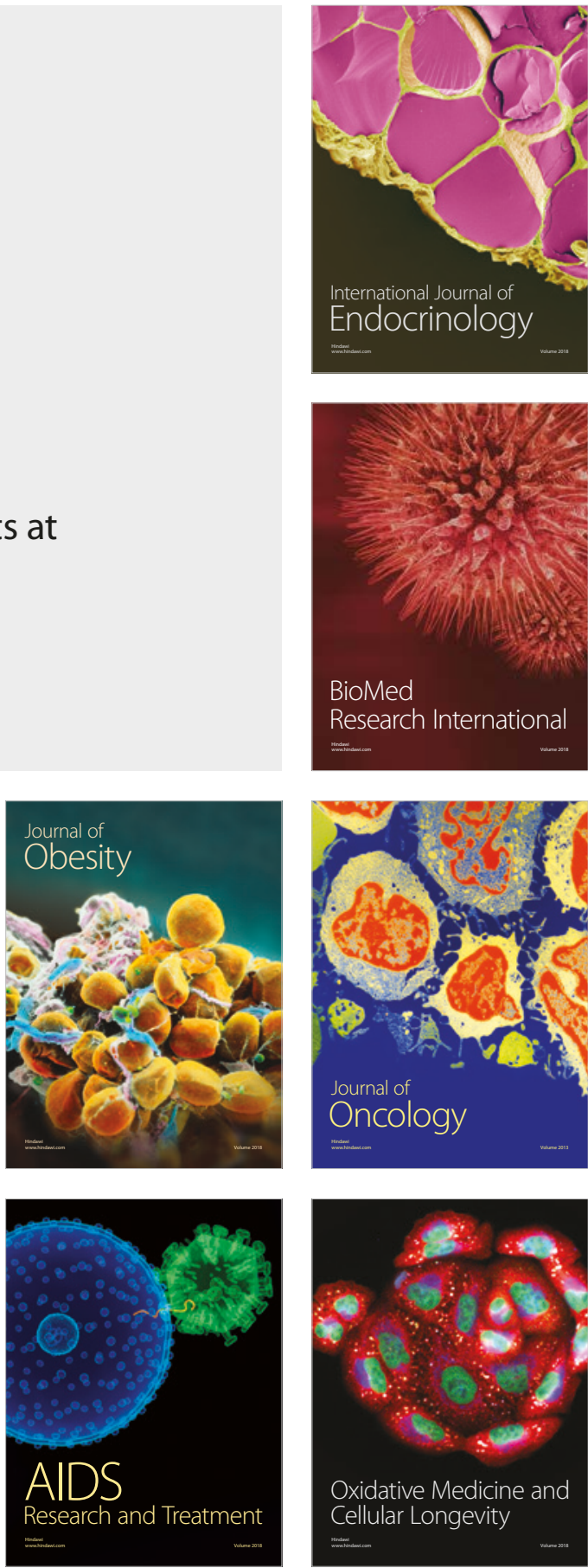